TRANSACTIONS OF THE

AMERICAN MATHEMATICAL SOCIETY

Volume 352, Number 1, Pages 209-242

S 0002-9947(99)02447-2

Article electronically published on September 8, 1999

\title{
A MULTIPLICATION IN CYCLIC HOMOLOGY
}

\author{
KIYOSHI IGUSA
}

\begin{abstract}
We define a multiplication on the cyclic homology of a commutative, cocommutative bialgebra $H$ with "superproduct." In the case when $H$ is a field of characteristic zero the cyclic homology becomes a polynomial algebra in one generator. (The Loday-Quillen multiplication is trivial in that case.)
\end{abstract}

In this paper we define an associative multiplication on the cyclic homology of certain commutative algebras $H$. The definition is based on the Cartesian product of sets instead of the shuffle product as in the case of [LQ] and [Ga]. When $H$ contains $\mathbb{Q}$ our product is compatible with the Hodge decomposition of $H C_{*}(H)$ so it has the form:

$$
H C_{n}^{(i)}(H) \otimes H C_{m}^{(j)}(H) \rightarrow H C_{n+m}^{(i+j)}(H) .
$$

The existence of this multiplication in the case $H=K$ is classical since it is just given by the tensor product of complex line bundle. However its significance came to our attention in joint work with John Klein as a consequence of our study of fiberwise Morse theory on circle bundles [IK] and in other joint works with R. Penner and R. Hain. By doing Morse theory on circles John Klein and I realized that there was a rationally nontrivial associative multiplication on the cyclic homology of the integral group ring on any cyclic group and that it had important consequences for pseudoisotopy theory. This will be explained in a later paper.

In this paper we show that this multiplication in cyclic homotopy is defined first in the case when the algebra is $H=K M=K[M]$ where $M$ is the underlying additive group of a ring, then in the case when $H$ is a Hopf algebra with "superproduct" in the sense of Gaucher [Ga] and finally in the more general case of any " $\mu$-algebra" (Example 12.4). In fact we do not need additive inverses in $M$ nor do we need an antipode for $H$ so we can take $M$ to be a monoid and $H$ to be a bialgebra. When $H=K$, i.e. $M=1$, the cyclic homology becomes a divided power algebra which is of course isomorphic to a polynomial algebra in one generator if $K$ is a field of characteristic zero.

We assume throughout the paper that $K$ is a commutative ring with 1 and that all tensor products are taken over $K$. This includes those tensor products which appear in the definition of cyclic homology. We also use Connes' original definition that a cyclic object in a category $\mathcal{C}$ is a covariant functor $\Lambda \rightarrow \mathcal{C}$ where $\Lambda$ is Connes' cyclic category. Since $\Lambda \approx \Lambda^{o p}$ this is the same as a contravariant functor $\Lambda \rightarrow \mathcal{C}$.

An outline of the paper is as follows. In the first section we give a topological definition of the multiplication in $H C_{*}(K M)$. The construction is so elementary that

Received by the editors April 14, 1994.

1991 Mathematics Subject Classification. Primary 18G60; Secondary 16W30.

This research was supported by NSF grant no. DMS 9002512.

(C)1999 American Mathematical Society 
it must have been noticed before. For example when $M=\{1\}$ the multiplication is induced by the map $B U(1) \times B U(1) \rightarrow B U(1)$ given by tensor product of universal line bundles. Coupled with the well-known isomorphism $H C_{*}(K) \approx H_{*}(B U(1) ; K)$ we get a multiplication on $H C_{*}(K)$ with all the properties that we have claimed.

In $\S \S 2-6$ we give an algebraic definition of this multiplication. The basic idea is as follows. Given generators $\left(x_{0}, \ldots, x_{n}\right)$ and $\left(y_{0}, \ldots, y_{m}\right)$ we get a "cylindrical" array of $(n+1)(m+1)$ elements $x_{i} * y_{i}$ (given a pairing $*$ on $M$ ). We take all maps $\phi$ of this cylinder into a circular array of $n+m+1$ points and multiply the elements $x_{i} * y_{j}$ which go to the same point. The sum of the resulting elements $\left(z_{0}, \ldots, z_{n+m}\right)$ with appropriate coefficients is the product of $\left(x_{0}, \ldots, x_{n}\right)$ and $\left(y_{0}, \ldots, y_{m}\right)$. The algebraic description has the advantage that it generalizes to bialgebras.

In $\S \S 7-9$ we verify some of the basic properties of the multiplication $\mu$. In $\S \S 10$ 13 we show that $\mu$ is compatible with the power operations of $[\mathrm{L}]$ and $[\mathrm{FT}]$. Finally, in the last five sections (14-18) we show that the algebraic definitions of $\mu$ and the power operations agree with the topological definitions given in section 1 in the case $H=K M$ when both are defined.

I would like to thank Tom Goodwillie for some very incisive comments on this research and for explaining to us his version of the power operations in cyclic homology. I would also like to thank J. Klein, R. Penner and R. Hain.

\section{A topological Description of the multiplication}

If $M$ is a multiplicative monoid then let $B M$ be the space of all configurations in $S^{1}$ consisting of a finite subset $Y$ of $S^{1}=U(1)$ together with a "labelling" function $\lambda: Y \rightarrow M \backslash 1$. We topologize $B M$ so that it is a CW complex with one $n$-cell for each $(n+1)$-tuple $x=\left(x_{0}, x_{1}, \ldots, x_{n}\right)$ in $M$ where $x_{1}, \ldots, x_{n}$ are not equal to 1 . The open cell $e_{x}$ corresponding to $x$ is the set of all configurations $(Y, \lambda)$ so that:

(a) $Y \backslash 1$ has exactly $n$ elements.

(b) $\lambda\left(y_{i}\right)=x_{i}$ for $1 \leq i \leq n$ where $y_{1}, \ldots, y_{n}$ are the elements of $Y \backslash 1$ in counterclockwise order.

(c) $1 \in Y$ if and only if $x_{0} \neq 1$.

(d) $\lambda(1)=x_{0}$ if $x_{0} \neq 1$.

As we go to the boundary of $e_{x}$ the elements of $Y$ either come together or go to $1 \in$ $S^{1}$. In the limiting configuration we should multiply the labels in counterclockwise order and delete points with resulting label equal to 1.

The space $B M$ together with its cell decomposition is the geometric realization of a simplicial set $F(M)$ given by $F_{n}(M)=M^{n+1}$ with face and degeneracy operations given by

$$
\begin{aligned}
\partial_{i}\left(x_{0}, \ldots, x_{n}\right) & =\left(x_{0}, \ldots, x_{i} x_{i+1}, \ldots, x_{n}\right) \text { for } 0 \leq i \leq n-1 \\
\partial_{n}\left(x_{0}, \ldots, x_{n}\right) & =\left(x_{n} x_{0}, x_{1}, \ldots, x_{n-1}\right) \\
s_{i}\left(x_{0}, \ldots, x_{n}\right) & =\left(x_{0}, \ldots, x_{i}, 1, x_{i+1}, \ldots, x_{n}\right) \text { for } 0 \leq i \leq n
\end{aligned}
$$

The homeomorphism $|F(M)| \cong B M$ is given by sending $(x, t) \in M^{n+1} \times \Delta^{n}$ to the configuration of points $Y=\left(y_{0}, \ldots, y_{n}\right)$ given by

$$
y_{i}=\exp \left(2 \pi \sqrt{-1}\left(t_{0}+\cdots+t_{i-1}\right)\right) \in S^{1}
$$

with labels $\lambda\left(y_{i}\right)=x_{i}$.

Let $G M$ be the space of all pairs $(C, X)$ where $C \in B U(1)$ and $X$ is a finite configuration in $C$ with labels in $M \backslash 1$. Since $C$ is a coset of $U(1)=S^{1}$ in the 
contractible topological abelian group $E U(1)$ it is an oriented circle. Then $G M=$ $B M \times_{U(1)} E U(1)$. Therefore for any commutative $\operatorname{ring} K$ we have: $H_{n}(G M ; K) \cong$ $H C_{n}(K M)$. (See [J], Theorem 3.1.)

Now suppose that the multiplicative monoid $M$ is commutative and has a bimultiplicative pairing $*: M \times M \rightarrow M$ so that $1 * x=x * 1=1$ for all $x \in M$. Then we obtain a continuous map $\mu: G M \times G M \rightarrow G M$ as follows.

$$
\mu((C, X),(D, Y))=(C D, X * Y)
$$

where $C D=\{c d \in E U(1) \mid c \in C, d \in D\} \in B U(1)$ and $X * Y$ is the configuration $\{x y \in C D \mid x \in X, y \in Y\}$ together with the corresponding labels $x * y$. We multiply the labels when points coincide.

If $*$ is associative or commutative then so is $\mu$. Also if $*$ has a two-sided neutral element $e$ then $\left(S^{1}, E\right)$ is a two-sided identity for $\mu$ where $E$ is the configuration in $S^{1}$ consisting of the one point $1 \in S^{1}$ with label $e$.

Theorem 1.1. If $*$ is associative with e then $H C_{*}(K M)$ becomes a graded ring. Furthermore

(a) This multiplication is natural with respect to maps $M \rightarrow M^{\prime}$ preserving multiplication, $*, 1$ and $e$ and with respect to change of rings $K \rightarrow K^{\prime}$.

(b) In the case $M=\{1\}=\{e\}, H C_{*}(K)$ is a divided power algebra, i.e. the product of the generators in degrees $a, b$ is given by $u_{a} u_{b}=\left(\begin{array}{c}a+b \\ a\end{array}\right) u_{a+b}$.

Proof. The homology ring $H_{*}(B U(1))$ is easy to compute and the answer is well known to be a divided power algebra.

Corollary 1.2. Suppose that $M$ is a finite group and $K$ is a field of characteristic 0 . Then the multiplication in $H C_{*}(K M)$ is given by $[x]_{i}[y]_{i}=\left(\begin{array}{c}i+j \\ i\end{array}\right)[x * y]_{i+j}$ where $[x]_{i}$ is the generator of $H C_{2 i}(K M)$ corresponding to $x \in M$.

Remark. If $(M, \cdot)$ is a group then $\langle M, \cdot, *\rangle$ is a ring and $K M$ is a ring object in the sense of Husemuller $[\mathrm{H}]$ or equivalently a Hopf algebra with superproduct in the sense of Gaucher [Ga].

Theorem 1.3. If $K$ contains $\mathbb{Q}$, then multiplication in $H C_{*}(K M)$ is compatible with the Hodge decomposition of $\mathrm{HC}_{*}(K M)$. Thus we get

$$
H C_{n}^{(i)}(K M) \otimes H C_{m}^{(j)}(K M) \rightarrow H C_{n+m}^{(i+j)}(K M) .
$$

Proof. The $k$-th power operation $1 / k \Psi^{k}=(-1)^{k-1} \lambda^{k}$ is given by the map $L^{k}$ : $G M \rightarrow G M$ given by $L^{k}(C, X)=\left(C^{k}, L^{k}(X)\right)$ where $C^{k} \in B U(1)$ is the $k$-fold product $C^{k}=C C \cdots C$ using the abelian group structure on $B U(1)$ and $L^{k}(X)$ is the configuration of points $\left(x_{i}\right)^{k} \in C^{k}$ given by the $k$-fold product in the abelian group $E U(1)$. The label on the point $z \in L^{k}(X)$ is the product of the labels of those points $x_{i} \in C$ for which $z=\left(x_{i}\right)^{k}$.

Since $C^{k} D^{k}=(C D)^{k}$ and $L^{k}(X) * L^{k}(Y)=L^{k}(X * Y)$ we see that the product operation $*$ strictly commutes with $L^{k}$ in the sense that

$$
L^{k}(C, X) * L^{k}(D, Y)=L^{k}(C D, X * Y)=L^{k}((C, X) *(D, Y)) .
$$

Consequently if $a \in H C_{n}^{(i)}(K M)$ and $b \in H C_{m}^{(j)}(K M)$ then $1 / k \Psi^{k}(a) * 1 / k \Psi^{k}(b)=$ $k^{i} a * k^{j} b=k^{i+j} a * b=1 / k \Psi^{k}(a * b)$ so $a * b \in H C_{n+m}^{(i+j)}(K M)$. 


\section{ACYCLIC CARRIERS}

Let $\left(C_{*}(K M), b\right)$ be the standard Hochschild complex given by $C_{n}(K M)=$ $K M^{\otimes(n+1)} \approx K M^{n+1}$ and $b\left(x_{0}, \ldots, x_{n}\right)=\left(x_{0} x_{1}, x_{2}, \ldots, x_{n}\right)-\left(x_{0}, x_{1} x_{2}, \ldots, x_{n}\right)+$ $\cdots+(-1)^{n}\left(x_{n} x_{0}, x_{1}, \ldots, x_{n-1}\right)$ and let $T(K M)$ be the total space of the following standard bicomplex whose homology is $H C_{*}(K M)$.

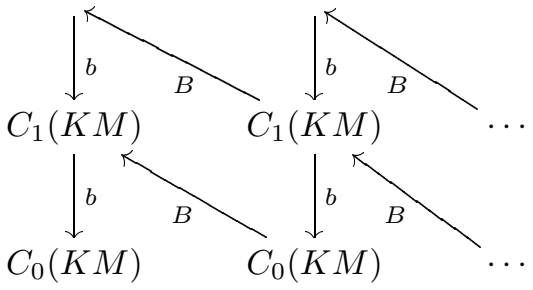

Here $B$ is given in a standard way by

$$
\begin{aligned}
B\left(x_{0}, \ldots, x_{n}\right)=\sum_{i=0}^{n}\left[(-1)^{n i}\left(1, x_{i}, \ldots, x_{n}, x_{0}, \ldots, x_{i-1}\right)\right. & \\
& \left.+(-1)^{n(i+1)}\left(x_{i-1}, 1, x_{i}, \ldots, x_{n}, x_{0}, \ldots, x_{i-2}\right)\right] .
\end{aligned}
$$

We will construct an acyclic carrier for a map $\mu: T(K M) \otimes T(K M) \rightarrow T(K M)$ which induces the multiplication on $H C_{*}(K M)$ defined in the last section. The purpose of this is to obtain (the existence of) an algebraic formula for this multiplication and to use this formula (without knowing what it is) to generalize the multiplication to the case of $H C_{*}(H)$ where $H$ is a bialgebra over $K$ with "superproduct." The purpose of this section is to describe the nature of the acyclic carrier that we will be using to specify both $\mu$ and the power operations $\Psi^{k}$.

If $C$ is a free $K$-complex then a basis for $C$ forms the set of objects of a category which we call a "basis category" for $C$.

Definition 2.1. A basis category (with coefficients in $K$ ) is defined to be a small category $\mathcal{B}$ together with a nonnegative grading on the set of objects deg: $\mathrm{Ob}(\mathcal{B}) \rightarrow$ $\mathbb{N}$ and an incidence coefficient $r_{\alpha} \in K$ for each morphism $\alpha: x \rightarrow y$ of degree $-1(\operatorname{deg}(\alpha)=\operatorname{deg}(y)-\operatorname{deg}(x))$ so that the following two conditions hold.

(1) For each object $x$ in $\mathcal{B}$ there are only finitely many morphisms $x \rightarrow y$ of degree -1 with nonzero incidence coefficient.

(2) For any morphism $\gamma$ in $\mathcal{B}$ of degree -2 we have $\sum r_{\beta} r_{\alpha}=0$ where the sum is taken over all factorizations $\gamma=\beta \alpha$ of $\gamma$ into a composition of two morphisms of degree -1 .

We say that $\mathcal{B}$ is a basis category for a free $K$-complex $C$ if $\mathcal{B}_{n}$ (the set of objects of $\mathcal{B}$ of degree $n$ ) is a free basis for $C_{n}$ and if the coefficient of $y \in \mathcal{B}_{n-1}$ in $\partial x$ for $x \in \mathcal{B}_{n}$ is equal to the sum $\sum r_{\alpha}$ of all incidence coefficients of all morphisms $\alpha: x \rightarrow y$. Since $C$ is uniquely determined by $\mathcal{B}$ we sometimes write $C=K \mathcal{B}$.

An augmentation $\varepsilon: C_{0} \rightarrow K$ for a free complex $C$ corresponds to an augmentation for the basis category $\mathcal{B}$. This is defined to be a system of coefficients $r_{b} \in K$ for each $b \in \mathcal{B}_{0}$ satisfying the obvious conditions similar to (2) above.

If $\mathcal{B}$ is an augmented basis category over $K$ and $D$ is an augmented $K$-complex then an acyclic carrier from $K \mathcal{B}$ to $D$ is defined to be a contravariant functor $C$ from $\mathcal{B}$ to the category of acyclic augmented complexes over $D$. An augmented chain 
map $f: K \mathcal{B} \rightarrow D$ is said to be carried by the carrier $C$ if for each $b \in \mathcal{B}_{n}, f(b) \in D_{n}$ lifts to some $\tilde{f}(b) \in C_{n}(b)$ so that, if $n \neq 0, \partial \tilde{f}(b)=\sum r_{\alpha} \alpha * \tilde{f}\left(t_{\alpha}\right)$ where the sum is taken over all morphisms $\alpha: b \rightarrow t_{\alpha}$ of degree -1 in $\mathcal{B}$ and $\varepsilon \tilde{f}(b)=r_{b}$ if $n=0$.

Theorem 2.2. Any acyclic carrier from $K \mathcal{B}$ to $D$ carries at least one chain map $K \mathcal{B} \rightarrow D$ and any two such maps are chain homotopic.

One standard example of a basis category is the opposite $\operatorname{simp}(X)^{o p}$ of the category of simplices of a simplicial set $X$. (See [W], p. 337.) The following related example comes from any cyclic set.

Let $X$ be a cyclic set, i.e. a covariant functor $\Lambda \rightarrow$ Sets where $\Lambda$ is Connes' cyclic category. Then we define the basic category $\mathcal{B}(X)$ in such a way that $K \mathcal{B}(X)$ is the total complex of the bicomplex $B_{*}(K X)$ as defined in [Go], II.2 where $K X$ is the cyclic free $K$-module generated by $X$.

Definition 2.3. For any cyclic set $X$ the basis category $\mathcal{B}(X)$ is given as follows.

(1) The objects of $\mathcal{B}(X)$ are all symbols $S_{i}(n, x)$ where $i \geq 0$ and $x \in X_{n}$.

(2) $\operatorname{deg}\left(S_{i}(n, x)\right)=n+2 i$.

(3) A morphism $S_{i}(n, x) \rightarrow S_{j}(m, y)$ is a morphism $\alpha:[n] \rightarrow[m]$ in $\Lambda$ so that $y=\alpha_{*} x$.

(4) The morphism $\partial_{i}: S_{j}(n, x) \rightarrow S_{j}\left(n-1, \partial_{i} x\right)$ has incidence coefficient $(-1)^{i}$.

(5) $s t^{k}: S_{i}(n, x) \rightarrow S_{i-1}\left(n+1, s t^{k} x\right)$ has incidence coefficient $(-1)^{n k}$. Here $s=t s_{n}$ so that $s\left(x_{0}, \ldots, x_{n}\right)=\left(1, x_{0}, \ldots, x_{n}\right)$.

(6) $t s t^{k}: S_{i}(n, x) \rightarrow S_{i-1}\left(n+1, t s t^{k} x\right)$ has incidence coefficient $(-1)^{n(k+1)}$.

(7) All other morphisms have coefficient 0.

(8) The augmentation of each element of the form $S_{0}(0, x)$ is 1.

As an example we take the cyclic set $M^{\Lambda}$ given by $\left(M^{\Lambda}\right)_{n}=M^{n+1}$ with the usual action of the cyclic operators: $\alpha_{\#}\left(x_{0}, \ldots, x_{n}\right)=\left(y_{0}, \ldots, y_{m}\right)$ where each $y_{j}$ is the product of $x_{i}$ for all $i \in \alpha^{-1}(j)$. Then $K \mathcal{B}\left(M^{\Lambda}\right)=T(K M)$.

A morphism of cyclic sets $X \rightarrow Y$ induces a morphism of basis categories $\mathcal{B}(X) \rightarrow \mathcal{B}(Y)$ is defined below and thus a chain map $K \mathcal{B}(X) \rightarrow K \mathcal{B}(Y)$.

Definition 2.4. A morphism of basis categories is a degree preserving functor $f: \mathcal{A} \rightarrow \mathcal{B}$ satisfying the following.

(a) For each object $X$ in $\mathcal{A}$ and each morphism $\beta: F(X) \rightarrow Y$ in $\mathcal{B}$ there is a unique object $Z$ in $\mathcal{A}$ and a unique morphism $\alpha: X \rightarrow Z$ in $\mathcal{A}$ so that $f(Z)=Y$ and $f(\alpha)=\beta$.

(b) $r_{\alpha}=f_{f(\alpha)}$ for all morphisms $\alpha$ in $\mathcal{A}$.

(c) $r_{X}=r_{f(X)}$ for all objects $X$ in $\mathcal{A}_{0}$.

It is easy to see that any morphism $f: \mathcal{A} \rightarrow \mathcal{B}$ induces an augmentation preserving chain map $f_{*}: K \mathcal{A} \rightarrow K \mathcal{B}$.

\section{ACYClic COMPLEX Given By a CyClicAlLy ORDERED SET}

Tony Elmendorf and perhaps others have been observed that Connes' category $\Lambda$ can be expressed in terms of cyclically ordered sets. In this section we construct an acyclic augmented complex $E(P)$ associated to any cyclically ordered set $P$. These will be used to construct acyclic carriers.

Definition 3.1. A cyclic partial ordering on a set $P$ is defined to be a partial ordering on the set $\widetilde{P}=P \times \mathbb{Z}$ so that: 
(a) $(x, n)<(x, n+1)$ for all $(x, n) \in \widetilde{P}$.

(b) $\sigma(x, n)=(x, n+1)$ gives an automorphism of the poset $\widetilde{P}$.

(c) For all $x, y \in P$ there is an $N \in \mathbb{Z}$ so that $(x,-N)<(y, 0)<(x, N)$.

We will sometimes use the notation $\sigma^{n}(x)=(x, n)$. To avoid confusion we should point out that the term "cyclic poset" already has a (different) meaning, namely it is a cyclic object in the category of posets.

Some important examples of cyclically ordered sets are as follows.

Definition 3.2. (a) $Z[n]=\{0,1,2, \ldots, n\}$ with the cyclic total ordering $0<1<$ $\cdots<n<\sigma(0)$.

(b) $\lambda^{k} Z[n]=\{0,1,2, \ldots, n\}$ with the cyclic partial ordering $0<1<\cdots<n<$ $\sigma^{k}(0)$.

(c) $Z[0,1)$ is the subset $[0,1)$ of $\mathbb{R}$ with the cyclic total ordering $(x, n)<(y, m)$ if $x+n<y+m$.

Definition 3.3. A morphism of cyclically ordered sets $\phi: P \rightarrow Q$ is defined to be an equivalence class of maps $\tilde{\phi}: \widetilde{P} \rightarrow \widetilde{Q}$ which are order preserving (i.e. $\tilde{\phi}(a) \leq \tilde{\phi}(b)$ whenever $a<b$ ) and which commutes with $\sigma$. Two such maps are equivalent if they differ by a power of $\sigma$. $\left(\tilde{\phi} \sim \tilde{\phi} \circ \sigma^{k}\right)$ For any morphism $\phi: P \rightarrow Q$ there is an induced set map $\bar{\phi}: P \rightarrow Q$ given by $\tilde{\phi}(x, n)=(\bar{\phi}(x), m)$.

Given two cyclically ordered set $P, Q$ the cartesian product $P \times Q$ has a product cyclic ordering given by $(x, y, n) \leq(p, q, m)$ if $(x, i) \leq(p, j)$ in $\widetilde{P}$ and $(y, n-i) \leq$ $(q, m-j)$ in $\widetilde{Q}$ for some $i, j$. Given morphisms $\phi: S \rightarrow P, \psi: T \rightarrow Q$ there is an induced morphism $\phi \times \psi: S \times T \rightarrow P \times Q$ given by $\widetilde{\phi \times \psi}(s, t, n)=(x, y, a+b+n)$ if $\tilde{\phi}(s, 0)=(x, a), \tilde{\psi}(t, 0)=(y, b)$. Although $P \times Q$ is not the categorical product of $P$ and $Q$ (e.g. there is no natural morphism $P \times Q \rightarrow P$ ) it does define a factor $\mathcal{C} \times \mathcal{C} \rightarrow \mathcal{C}$ where $\mathcal{C}$ is the category of cyclically ordered sets.

A morphism $\alpha: Z[n] \rightarrow Z[m]$ can be represented by a sequence of words $w_{0}, w_{1}, \ldots, w_{m}$ in the letters $0,1, \ldots, n$ so that the word $w_{0} w_{1} \cdots w_{m}$ is equal to the word $(i)(i+1) \cdots(n)(0) \cdots(i-1)$ for some $i$. The correspondence is given by letting $w_{j}$ be the product of the elements of $\bar{\alpha}^{-1}(j)$ in the correct order. It should be well known that the objects $Z[n]$ together with the morphisms between them give Connes' category $\Lambda$.

To obtain the correct names for cyclic operators (morphisms induced by covariant functors on $\Lambda$ ) we make the following notation.

Definition 3.4. In the above notation the morphisms $\partial_{i}, s_{i}, s, t$ are given by:

(a) $\partial_{i}=(0,1, \ldots, i(i+1), \ldots, n): Z[n] \rightarrow Z[n-1]$ if $0 \leq i \leq n-1$,

(b) $\partial_{n}=(n 0,1, \ldots, n-1): Z[n] \rightarrow Z[n-1]$,

(c) $s_{i}=(0,1, \ldots, i, \varnothing, i+1, \ldots, n): Z[n] \rightarrow Z[n+1]$ where $\varnothing$ is the empty word,

(d) $t=(n, 0,1, \ldots, n-1): Z[n] \rightarrow Z[n]$,

(e) $s=t s_{n}=(\varnothing, 0,1, \ldots, n): Z[n] \rightarrow Z[n+1]$.

For any cyclically ordered set $P$ let $X(P)$ denote the cyclic set given by $X_{n}(P)=$ $\operatorname{Hom}(P, Z[n])$. If we represent and element $\phi$ of $\operatorname{Hom}(P, Z[n])$ in the format 
$\left\langle a_{0}, \ldots, a_{n}\right\rangle$ where $a_{i}=\tilde{\phi}^{-1}(i)$ then the cyclic operators act on $X(P)$ in the expected way by

$$
\begin{aligned}
& \partial_{i}\left\langle a_{0}, \ldots, a_{n}\right\rangle=\left\langle a_{0}, \ldots, a_{j} \cup a_{i+1}, \ldots, a_{n}\right\rangle \text { for } 0 \leq i \leq n-1, \\
& \partial_{n}\left\langle a_{0}, \ldots, a_{n}\right\rangle=\left\langle\sigma^{-1} a_{n}, a_{0}, \ldots, a_{n-1}\right\rangle, \\
& s_{i}\left\langle a_{0}, \ldots, a_{n}\right\rangle=\left\langle a_{0}, \ldots, a_{i}, \varnothing, a_{i+1}, \ldots, a_{n}\right\rangle \text { for } 0 \leq i \leq n, \\
& s\left\langle a_{0}, \ldots, a_{n}\right\rangle=\left\langle\varnothing, a_{0}, \ldots, a_{n}\right\rangle, \\
& t\left\langle a_{0}, \ldots, a_{n}\right\rangle=\left\langle\sigma^{-1} a_{n} \cup a_{0}, \ldots, a_{n-1}\right\rangle .
\end{aligned}
$$

More generally, for any morphism $\psi: Z[n] \rightarrow Z[m]$ we have

$$
\psi_{*} \phi=\left\langle\tilde{\phi}^{-1} \tilde{\psi}^{-1}(0), \ldots, \tilde{\phi}^{-1} \tilde{\psi}^{-1}(m)\right\rangle .
$$

We define $E(P)$ to be the augmented complex $E(P)=K \mathcal{B} X(P)$. It is clear from the definitions that $X, E$ are contravariant functors from the category of cyclically ordered sets to the category of cyclic sets or augmented $K$-complexes respectively. Since the augmented complex $E(P)$ plays a crucial role in the rest of the paper we will describe it explicitly.

Proposition 3.5. (1) The objects of the basis category $\mathcal{B} X(P)$ are symbols of the form $s_{a}(n, \phi)$ where $\phi: P \rightarrow Z[n]$ is a morphism and $a, n \geq 0$.

(2) A morphism $\alpha: S_{a}(n, \phi) \rightarrow S_{b}(m, \psi)$ is given by $\alpha: Z[n] \rightarrow Z[m]$ so that $\psi=\alpha \circ \phi$.

(3) In $E(P)$ we have

$$
\begin{aligned}
d S_{a}(n, \phi)=\sum_{i=0}^{n}\left[(-1)^{i} S_{a}\left(n, \partial_{i} \phi\right)\right. & \\
& \left.+(-1)^{n i} S_{a-1}\left(n+1, s t^{i} \phi\right)+(-1)^{n i+n} S_{a-1}\left(n+1, t s t^{i} \phi\right)\right]
\end{aligned}
$$

(i.e. $d=b+B)$ where the undefined terms $S_{-1}(*, *)$ are taken to be zero.

Remark. We will sometimes abbreviate this expression for $d S_{a}(n, \phi)$ as

$$
\sum_{\alpha \in W(a, n)} \operatorname{sgn}(\alpha) S_{*}(*, \alpha \phi)
$$

where $W(a, n)$ is the set of all operations $\partial_{i}, s t^{i}, t s t^{i}$ appearing in the above sum.

The rest of this section is devoted to the proof of the following theorem.

Theorem 3.6. $E(P)$ is acyclic for any nonempty finite cyclically ordered set $P$.

Proof. From the topological viewpoint this is more or less obvious.

Let $M(P)$ be the space of all maps $f: \widetilde{P} \rightarrow \mathbb{R}$ so that

(1) $f \circ \sigma(x)=f(x)+1$ for all $x \in \widetilde{P}$,

(2) $f(x) \leq f(y)$ if $x<y$ in $\widetilde{P}$.

Then $M(P)$ is convex and thus contractible. (By Lemma 3.7 below $M(P)$ is nonempty.) This means that the space of all morphisms $P \rightarrow Z[0,1)$ is homotopy equivalent to $M(P) / \mathbb{Z} \simeq S^{1}=U(1)$ and so $(M(P) / \mathbb{Z}) \times_{U(1)} E U(1)$ is contractible. But $H_{*} E(P)$ is the homology of this space with coefficients in $K$ so $E(P)$ is acyclic. An algebraic version of this proof is given below.

Lemma 3.7. If $P$ is a finite cyclically ordered set then there is at least one morphism $P \rightarrow Z[n]$ for each $n$. 
Proof. It suffices to take $n=0$ since $Z[0]$ maps to $Z[n]$ for all $n$.

If $P$ is empty or contains only one element then there is a unique morphism $P \rightarrow Z[0]$ so suppose that $P$ has at least two elements and the lemma hold for $S=P \backslash x$ where $x \in P$ is fixed. Let $f: \widetilde{S} \rightarrow \mathbb{Z}$ be a poset map representing a morphism $S \rightarrow Z[0]$. Then we can extend $f$ to a poset map $g: \widetilde{P} \rightarrow \mathbb{Z}$ by letting $g(x, n)$ be the smallest value of $f(y, m)$ as $(y, m)$ ranges over all elements of $\widetilde{S}$ greater than $(x, n)$.

The algebraic proof of Theorem 3.6 starts with a special case.

Lemma 3.8. $E(Z[m])$ is acyclic for all $m \geq 0$.

Proof. Since $E(Z[m])$ is a free $K$-complex with integer coefficients it is chain contractible in general if and only if it is contractible in the special case when $K=\mathbb{Z}$. This in turn is equivalent to the condition that $E(Z[m]) \otimes F$ is acyclic for any field $F$. Thus it suffices to prove the lemma in the case when $K$ is a field.

The proof will be by induction on $m$ using the cyclic homology of monomial relation algebras over a field $K$. (See [IZ].) Suppose first that $m=0$. Then $E(Z[0])$ is isomorphic to the weight $x$ component of the total complex $T(K[x])$ of the polynomial algebra $K[x]$. The lemma in this case follows from an easy calculation starting with the observation that the only nondegenerate terms in $C_{*}(K[x])$ of weight $x$ are $x \in C_{0}(K[x])$ and $1 \otimes x \in C_{1}(K[x])$.

Now suppose that $m \geq 1$ and that the lemma holds for $m-1$. Let $M_{m}$ be the free associative monoid generated by the symbols $x_{0}, x_{1}, \ldots, x_{m}$, i.e. $M_{m}$ is the set of all words in these symbols. If we define an equivalence relation on $M_{m}$ by saying that $a, b \in M_{m}$ are cyclically equivalent when the word $b$ is a cyclic permutation of the word $a$ then the cyclic homology of $K M_{n}$ decomposes as $H C_{*}\left(K M_{m}\right)=$ $\bigoplus_{c} H C_{*}\left(K M_{m}\right)^{c}$ where $c$ runs over all cyclic equivalence classes in $M_{m}$. This is because the Hochschild complex $C_{*}\left(K M_{m}\right)$ and the cyclic complex $T\left(K M_{m}\right)$ decomposes in the same way.

If we compare the definitions we see that the complex $E(Z[m])$ is isomorphic to the complex $T\left(K M_{m}\right)^{c(m)}$ where $c(m)$ is the cyclic equivalence class of $x_{0} x_{1} \cdots x_{m}$. However there is a short exact sequence:

$$
0 \rightarrow T\left(K M_{m-1}\right)^{c(m-1)} \rightarrow T\left(K M_{m}\right)^{c(m)} \rightarrow T\left(K M_{m} / J\right)^{c(m)} \rightarrow 0
$$

where the first map is indicated by the morphism $f: M_{m-1} \rightarrow M_{m}$ given by $f\left(x_{i}\right)=x_{i+1}$ for $i<m-1$ and $f\left(x_{m-1}\right)=x_{m} x_{0}$ and the second map is the natural quotient map where $J$ is the two-sided ideal in $K M_{m}$ generated by all monomials of the form $x_{i} x_{j}$ where $j \leq i$. Therefore by induction on $m$ it suffices to show that $T\left(K M_{m} / J\right)^{c(m)}$ is acyclic as a nonaugmented complex, i.e. that $H C_{*}\left(K M_{m} / J\right)^{c(m)}=0$.

Since $J$ is generated by monomials in the noncommuting generators $x_{0}, \ldots, x_{m}$ and since $c(m)=x_{0} x_{1} \cdots x_{m}$ is aperiodic we may use [IZ], Lemma 4.8 which implies that $H C_{*}\left(K M_{m} / J\right)^{c(m)} \approx H_{*}(C X, X ; K)$ where $C X$ is the cone of the simplicial complex $X(C X=*$ if $X$ is empty) and $X$ is the finite simplicial with vertices given by all minimal "relations" (i.e. generators of $J$ ) which are subwords of words in the cyclic class $c(m)$. In this case $X$ has only one vertex $x_{m} x_{0}$ so we are done. However for the sake of completeness we give the general formula for the higher simplices of $X$. The vertices $w_{0}, w_{1}, \ldots, w_{k}$ in $X$ span a $k$-simplex in $X$ if and only if they all occur as subwords of the same word $x_{i} x_{i+1} \cdots x_{m} x_{0} \cdots x_{i-1} \in c(m)$. 
Algebraic proof of Theorem 3.6. We will do induction on the complexity of $P$ which is defined to be the number of elements $(x, y, k)$ in $P \times P \times \mathbb{Z}$ so that $(x, 0)$ and $(y, k)$ are unrelated in the poset $\widetilde{P}$. By (c) in Definition 3.1 the integer $k$ is bounded and thus the complexity if finite.

The complexity of $P$ is zero if and only if $P$ is isomorphic to some $Z[m]$. By Lemma 3.8 the theorem holds in this case. If the complexity of $P$ is nonzero then $\widetilde{P}$ contains two unrelated elements $a, b$. Let $P_{+}, P_{-}$be the cyclically ordered sets obtained from $P$ by adding the condition that $a<b, b<a$ respectively. Let $P_{0}$ be the cyclically ordered sets obtained from $P$ by coalescing $a, b$. Then $E(P)=$ $E\left(P_{+}\right)+E\left(P_{-}\right)$and $E\left(P_{0}\right) \approx E\left(P_{+}\right) \cap E\left(P_{-}\right)$. Since $P_{+}, P_{-}, P_{0}$ have smaller complexity than $P$ the complexes $E\left(P_{+}\right), E\left(P_{-}\right), E\left(P_{0}\right)$ are acyclic by induction. Therefore $E(P)$ is acyclic.

\section{The multiplication $\mu$ ON $H C_{*}(K M)$}

Since $\mathcal{B}\left(M^{\Lambda}\right)$ is a basis category for the free complex $T(K M)$ the product category $\mathcal{B}\left(M^{\Lambda}\right) \times \mathcal{B}\left(M^{\Lambda}\right)$ with the appropriate sign conventions for the incidence coefficients will be a basis category for $T(K M) \otimes T(K M)$. Therefore we need to construct an acyclic complex over $T(K M)$ for each object of $\mathcal{B}\left(M^{\Lambda}\right) \times \mathcal{B}\left(M^{\Lambda}\right)$ and show that it has the required properties. We assume that $M$ is a commutative monoid and that $*: M \times M \rightarrow M$ is a bimultiplicative map so that $1 * x=x * 1=1$ for all $x \in M$, i.e. * distributes over any finite product in $M$ including the empty product 1 .

If $x=\left(x_{0}, \ldots, x_{n}\right), y=\left(y_{0}, \ldots, y_{m}\right)$ which we consider as set maps $x: Z[n] \rightarrow$ $M, y: Z[m] \rightarrow M$ then $x * y$ is defined to be the set map $Z[n, m] \rightarrow M$ which sends $(i, j)$ to $x_{i} * y_{j}$. (We use an abbreviation $Z[n, m]=Z[n] \times Z[m]$ since this product occurs quite often.) Given any morphism $\phi: Z[n, m] \rightarrow Z[k]$ let $(x * y)^{\#} \phi=\phi_{\#}(x * y)=\left(z_{0}, \ldots, z_{k}\right) \in M^{k+1}$ where $z_{t}=\prod\left\{x_{i} * y_{j} \mid \bar{\phi}(i, j)=t\right\}$ is the product in $M$ of the images under $x * y$ of the elements of $\bar{\phi}^{-1}(t) \subseteq Z[n, m]$. Since $M$ is commutative with unit (for the empty product) this is well defined and $(x * y)^{\#}$ gives a morphism of cyclic sets $X Z[n, m] \rightarrow M^{\Lambda}$. Let $D(x * y)$ be the augmented $K$-complex $E(Z[n, m])=K \mathcal{B}(X Z[n, m])$ together with the chain map to $T(K M)=K \mathcal{B}\left(M^{\Lambda}\right)$ induced by the cyclic map $(x * y)^{\#}$.

In order to be an acyclic carrier $D$ needs to be a contravariant functor from $\mathcal{B}\left(M^{\Lambda}\right) \times \mathcal{B}\left(M^{\Lambda}\right)$ into the category of acyclic complexes over $T(K M)$. So suppose that $\alpha \times \beta:\left(S_{*}(n, x), S_{*}(m, y)\right) \rightarrow\left(S_{*}\left(\alpha, \alpha_{\#} x\right), S_{*}\left(b, \beta_{\#} y\right)\right)$ is a morphism in $\mathcal{B}\left(M^{\Lambda}\right) \times \mathcal{B}\left(M^{\Lambda}\right)$ given by two cyclic operators $\alpha: Z[n] \rightarrow Z[a], \beta: Z[m] \rightarrow Z[b]$. (We ignore the irrelevant subscripts in $S_{*}$.) Then $\alpha, \beta$ give a morphism $\alpha \times \beta$ : $Z[n, m] \rightarrow Z[a, b]$ which induces a morphism

$$
\operatorname{Hom}(Z[a . b], Z[k]) \rightarrow \operatorname{Hom}(Z[n, m], Z[k]) .
$$

This gives a morphism of cyclic sets $(\alpha \times \beta)^{*}: X Z[a, b] \rightarrow X Z[n, m]$.

Lemma 4.1. The following diagram commutes, i.e.

$$
\left(\alpha_{\#} x * \beta_{\#} y\right)^{\#}=(x * y)_{\#}(\alpha \times \beta)^{*}:
$$




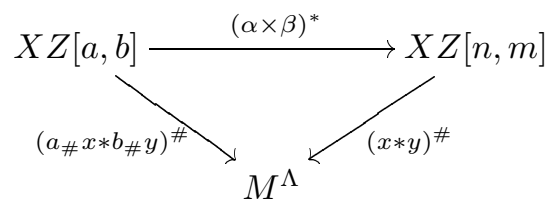

Proof. Take $\phi: Z[a, b] \rightarrow Z[k]$ in $X_{k} Z[a, b]$. Then

$$
\begin{aligned}
(x * y)^{\#}(\alpha \times \beta)^{*}(\phi) & =(x * y)^{\#}(\phi \circ(\alpha \times \beta)) \\
& =(\phi \circ(\alpha \times \beta))_{\#}(x * y)=\phi_{\#}(\alpha \times \beta)_{\#}(x * y) \\
& =\phi_{\#}\left(\alpha_{\#} x * \beta_{\#} y\right)=\left(\alpha_{\#} x * \beta_{\#} y\right)^{\#}(\phi)
\end{aligned}
$$

where the crucial step $(\alpha \times \beta)_{\#}(x * y)=\alpha_{\#} x * \beta_{\#} y$ follows from the definition of $\alpha_{\#} x, \beta_{\#} y$ given at the end of $\S 2$ and the fact that $*$ distributes over multiplication.

Lemma 4.1 implies that $(\alpha \times \beta)^{*}: D\left(\alpha_{\#} x * \beta_{\#} y\right)=E(Z[a, b]) \rightarrow E(Z[n, m])=$ $D(x * y)$ is a morphism of complexes over $T(K M)$.

Definition 4.2. Let $\mu: T(K M) \otimes T(K M) \rightarrow T(K M)$ be a chain map carried by the acyclic carrier $D$.

\section{A "FORMULA" FOR $\mu$}

In order to show that the multiplication $\mu$ can be extended to the case of a bialgebra $H$ with "superproduct" and to prove many of the properties of $\mu$ we need a formula for $\mu$ satisfying certain properties. In this section we prove only the existence of such a formula.

We note that if $\mu$ is carried by the carrier $D$ of the previous section then for each object $\left(S_{a}(n, x), S_{b}(m, y)\right)$ of $\mathcal{B}\left(M^{\Lambda}\right) \times \mathcal{B}\left(M^{\Lambda}\right)$ we have an element

$$
\tilde{\mu}\left(S_{a}(n, x), S_{b}(m, y)\right) \in D_{k}(x * y)=E(Z[n, m])
$$

where $k=n+m+2 a+2 b$ which is compatible with augmentation and boundary operators in a certain way, for example $\tilde{\mu}\left(S_{0}(0, x), S_{0}(0, y)\right) \in E_{0}(x * y) \cong K$ must have augmentation equal to $1 \in K$.

If these elements (1) depend only on $a, b, n, m$ (i.e. are independent of $x, y$ ) then we get a "uniform family" of elements $F_{a b}(n, m) \in E_{k}(Z[n, m])$ for $\mu$ which gives the following uniform formula for $\mu$.

$$
\mu\left(S_{a}(n, x), S_{b}(m, y)\right)=(x * y)^{\#} F_{a b}(n, m) .
$$

A family of elements $F_{a b}(n, m)$ is a uniform family for $\mu$ if it satisfies certain conditions which we will give below. Any two such families will be "uniformly homotopic" since $E(Z[n, m])$ is acyclic.

Definition 5.1. By a uniform family for the multiplication $\mu$ we mean a sequence of elements $F_{a b}(n, m) \in E_{k}(Z[n, m])$ where $k=n+m+2 a+2 b$ for all $n, m, a, b \geq 0$ satisfying the following conditions.

(a) $F_{00}(0 m 0) \in E_{0}(Z[0,0])$ is the unique element with augmentation 1. 
(b)

$$
\begin{aligned}
d F_{a b}(n, m)= & \sum_{i=0}^{n}\left[(-1)^{i}\left(\partial_{i} \times 1\right)^{*} F_{a b}(n-1, m)\right. \\
& +(-1)^{n i}\left(s t^{i} \times 1\right)^{*} F_{a-1, b}(n+1, m) \\
& \left.+(-1)^{n i+n}\left(t s t^{i} \times 1\right)^{*} F_{a-1, b}(n+1, m)\right] \\
& +(-1)^{n} \sum_{j=0}^{m}\left[(-1)^{j}\left(1 \times \partial_{j}\right)^{*} F_{a b}(n, m-1)\right. \\
& \quad+(-1)^{m j}\left(1 \times s t^{j}\right)^{*} F_{a, b-1}(n, m+1) \\
& \left.\quad+(-1)^{m j+m}\left(1 \times t s t^{j}\right)^{*} F_{a, b-1}(n, m+1)\right] .
\end{aligned}
$$

The undefined terms in (b) which occur when $a, b, n$ or $m=0$ are understood to be zero. Using the terminology of the remark following Proposition 3.5 the right-hand side of (b) can be written as:

$$
\begin{aligned}
& \sum_{\alpha \in W(a, n)} \operatorname{sgn}(\alpha)(\alpha \times 1)^{*} F_{* b}(*, m) \\
& \quad+(-1)^{n} \sum_{\beta \in W(b, m)} \operatorname{sgn}(\beta)(1 \times \beta)^{*} F_{a^{*}}(n, *) .
\end{aligned}
$$

We also use the expression $F d_{a b}(n, m)$ to denote the right-hand side of (b).

Proposition 5.2. Let $F$ be a uniform family for $\mu$ then

(a) The expression (2) defines a chain map $\mu=\mu_{F}: T(K M) \otimes T(K M) \rightarrow$ $T(K M)$ carried by $D$.

(b) $\mu_{F}$ is natural with respect to morphisms of monoids $M \rightarrow N$.

Proposition 5.3. A uniform family $F$ for $\mu$ exists. Furthermore $F$ can be chosen so that

(a) $F_{a 0}(n, 0)=S_{a}\left(n, p_{1}\right)$ for all $a, n \geq 0$ where $p_{1}: Z[n, 0] \rightarrow Z[n]$ is the natural isomorphism.

(b) $F_{0 b}(0, m)=S_{b}\left(m, p_{2}\right)$ for all $b, m \geq 0$ where $p_{2}: Z[0, m] \rightarrow Z[m]$ is the natural isomorphism.

Proof. Take the special case $M=\{1\}$ and let $\mu_{1}: T(K) \otimes T(K) \rightarrow T(K)$ be a chain map carried by an acyclic carrier $D$. Then $F_{a b}(n, m)=\tilde{\mu}_{1}\left(S_{a}(n, i d), S_{b}(m, i d)\right)$ is the desired family of elements. (Definition 5.1 is equivalent to the condition that $\mu_{1}$ is carried by $D$.) The elements $F_{a b}(n, m)$ can be constructed by induction on the bidegree $(n+2 a, m+2 b)$. We can start with formulas (a), (b). The first nontrivial case if $F_{00}(1,1)$.

Definition 5.4. By a uniform homotopy between two uniform families $F, F^{\prime}$ we mean a homotopy between the corresponding chain maps $T(K) \otimes T(K) \rightarrow T(K)$ which is carried by $D$.

Proposition 5.5. A uniform homotopy $G: F \simeq F^{\prime}$ is given by a family of elements $G_{a b}(n, m) \in E_{k+1}(Z[n, m])$ satisfying the following.

(a) $d G_{00}(0,0)=0$. 
(b) $F_{a b}(n, m)-F_{a b}^{\prime}(n, m)=d G_{a b}(n, m)+G d_{a b}(n, m)$ where

$$
\begin{aligned}
G d_{a b}(n, m)= & \sum_{\alpha \in W(a, n)} \operatorname{sgn}(\alpha)(\alpha \times 1)^{*} G_{* b}(*, m) \\
& +(-1)^{n} \sum_{\beta \in W(b, m)} \operatorname{sgn}(\beta)(1 \times \beta)^{*} G_{a *}(n, *) .
\end{aligned}
$$

Proposition 5.6. Let $\mu, \mu^{\prime}: T(K M) \otimes T(K M) \rightarrow T(K M)$ be given by the uniform families $F, F^{\prime}$ and let $G: F \simeq F^{\prime}$ be a uniform homotopy. Then there is a homotopy $g: \mu \simeq \mu^{\prime}$ carried by $D$ and this homotopy is given by:

$$
\begin{gathered}
\tilde{g}\left(S_{a}(n, x), S_{b}(m, y)\right)=G_{a b}(n, m), \\
g\left(S_{a}(n, x), S_{b}(m, y)\right)=(x * y)^{\#} G_{a b}(n, m) .
\end{gathered}
$$

\section{Extension of $\mu$ TO BIALGEBRAS}

We will define a multiplication on the cyclic homology of a commutative, cocommutative $K$-bialgebra with "superproduct." But first we recall the definitions. A $K$-bialgebra $H=(H, m, u, \Delta, \varepsilon)$ is a $K$-algebra $(H, m, u)$ together with a coalgebra structure $(H, \Delta, \varepsilon)$ so that the diagonal (comultiplication) $\Delta: H \rightarrow H \otimes H$ and augmentation map $\varepsilon: H \rightarrow K$ are morphisms of $K$-algebras. (See, e.g., [S] for details.) In particular we have

$$
\begin{aligned}
& \Delta(x y)=\Delta(x) \Delta(y)=\sum_{(x),(y)} x_{(1)} y_{(1)} \otimes x_{(2)} y_{(2)} \quad\left(\text { or } "(x y)_{(i)}=x_{(i)} y_{(i)} "\right), \\
& \Delta(1)=1 \otimes 1 \text { where } 1=u(1), \\
& \varepsilon(x, y)=\varepsilon(x) \varepsilon(y), \\
& \varepsilon(1)=1 .
\end{aligned}
$$

Any monoid algebra $K M$ has the structure of a $K$-bialgebra given by $\Delta\left(\sum a_{i} x_{i}\right)$ $=\sum a_{i} x_{i} \otimes x_{i}, \varepsilon\left(\sum a_{i} x_{i}\right)=\sum a_{i}$. This comultiplication is obviously cocommutative.

Definition 6.1 ([Ga]). Suppose that $H$ is a $K$-bialgebra. Then by a superproduct on $H$ we mean a $K$-linear map $*: H \otimes H \rightarrow H$ satisfying the following for all $x, y, z \in H$.

(a) $\Delta(x * y)=\Delta(x) * \Delta(y)$, i.e., " $(x * y)_{(i)}=x_{(i)} * y_{(i)}$."

Here $*:(H \otimes H) \otimes(H \otimes H) \rightarrow H \otimes H$ is given by $(a \otimes b) *(c \otimes d)=(a * c) \otimes(b * d)$,

(b) $\varepsilon(x * y)=\varepsilon(x) \varepsilon(y)$,

(c) $x * 1=1 * x=u \varepsilon(x)$,

(d) (left distributivity) $x *(y z)=\sum_{(x)}\left(x_{(1)} * y\right)\left(x_{(2)} * z\right)$,

(e) (right distributivity) $x y * z=\sum_{(z)}\left(x * z_{(1)}\right)\left(y * z_{(2)}\right)$.

Note that any bimultiplicative map $*: M \times M \rightarrow M$ on a monoid $M$ satisfying $x * 1=1 * x=1$ gives a superproduct on $K M$.

Definition $6.2([\mathrm{Ga}])$. A unit for $(H, *)$ is an element $e$ of $H$ satisfying the following.
(a) $\Delta(e)=e \otimes e$.
(b) $\varepsilon(e)=1$.
(c) $x * e=e * x=x$ for all $x \in H$. 
Note that if $*$ is associative with unit $e$, then conditions $6.1(\mathrm{a}),(\mathrm{b})$ and $6.2(\mathrm{a})$, (b), (c) are equivalent to saying that $(H, *, e, \Delta, \varepsilon)$ is a bialgebra.

Given a commutative, cocommutative $K$-bialgebra $H$ with superproduct $*$ and a uniform family $F$ we would like to construct a chain map $\mu: T(H) \otimes T(H) \rightarrow T(H)$ with the formula

$$
\mu\left(S_{a}(n, x), S_{b}(m, y)\right)=(x * y)^{\#} F_{a b}(n, m)
$$

if $x \in C_{n}(H)=H^{\otimes(n+1)}$ and $y \in C_{m}(H)=H^{\otimes(m+1)}$. In order for this to be defined we need is a chain map $(x * y)^{\#}: E(Z[n, m]) \rightarrow T(H)$.

Given $x=\left(x^{0}, x^{1}, \ldots, x^{n}\right) \in H^{n+1}, y=\left(y^{0}, y^{1}, \ldots, y^{m}\right) \in H^{m+1}$ let $x * y$ denote the element of $H^{\otimes(n+1)(m+1)}$ given by the $(n+m+2)$-fold iterated sum

$$
x * y=\sum_{\left(x^{0}\right)} \cdots \sum_{\left(x^{n}\right)} \sum_{\left(y^{0}\right)} \cdots \sum_{\left(y^{m}\right)}\left(x_{(j)}^{i} * y_{(i)}^{j}\right)
$$

where we use the standard notation $\sum_{\left(x^{i}\right)}\left(x_{(0)}^{i}, \ldots, x_{(m)}^{i}\right)$ for the $m$-fold diagonal of $x^{i}$ and similarly for $y^{j}$.

Lemma 6.3. (a) $x * y$ is $K$-bilinear in $x, y$ and is therefore defined for any $x \in$ $H^{\otimes(n+1)}$ and $y \in H^{\otimes(m+1)}$.

(b) Given morphisms $\alpha: Z[n] \rightarrow Z[a], \beta: Z[m] \rightarrow Z[b]$ we have $\left(\alpha_{\#} x\right) *\left(\beta_{\#} y\right)=$ $(\alpha \times \beta)_{\#}(x * y)$ where $\left(\phi_{\#} z\right)^{j}=\prod\left\{z^{i} \mid \bar{\phi}(i)=j\right\}$ for any $\phi: P \rightarrow Q$ and $z \in H^{\otimes P}$.

Proof. This follows from the cocommutativity of $H$ and the fact that $*$ distributes over multiplication. (When $\alpha, \beta$ are not surjective we also need conditions (b), (c) of 6.1.$)$

Given any element $\left(z^{i j}\right)$ of $H^{(n+1)(m+1)}$ we have an induced chain map $\left(z^{i j}\right)^{\#}$ : $E(Z[n, m]) \rightarrow T(H)$ given on the generators $S_{a}(b, \phi: Z[n, m] \rightarrow Z[b])$ by

$$
\left(z^{i j}\right)^{\#} S_{a}(b, \phi)=S_{a}\left(b, \phi_{\#}\left(z^{i j}\right)\right)=S_{a}\left(b,\left(c^{0}, \ldots, c^{b}\right)\right)
$$

where each $c^{k}$ is given by $c^{k}=\prod\left\{z^{i j} \mid \bar{\phi}(i, j)=k\right\}$. Since $H$ is commutative with unit this is well-defined. The map $\left(z^{i j}\right)^{\#}$ commutes with all cyclic operators (it is a morphism of cyclic $K$-modules) and therefore induces a chain map as required. Since $\left(z^{i j}\right)^{\#}$ is $K$-multilinear the definition extends to any element of the tensor product $H^{\otimes(n+1)(m+1)}$. Thus $(x * y)^{\#}: E(Z[n, m]) \rightarrow T(H)$ is defined.

Proposition 6.4. The expression (5) defines an augmented chain map $\mu: T(H) \otimes$ $T(H) \rightarrow T(H)$.

Proof. We compare $d \mu, \mu d$ using the definition of $F$.

$$
\begin{aligned}
& d \mu\left(S_{a}(n, x), S_{b}(m, y)\right)=(x * y)^{\#} d F(n, m)=\sum \pm(x * y)^{\#}(\alpha \times \beta)^{*} F^{\prime}, \\
& \mu d\left(S_{a}(n, x), S_{b}(m, y)\right)=\sum \pm\left(\alpha_{\#} x * \beta_{\#} y\right)^{\#} F^{\prime} .
\end{aligned}
$$

But as in Lemma 4.1 we have the formal identity $(x * y)^{\#}(\alpha \times \beta)^{*}=\left[(\alpha \times \beta)_{\#}(x * y)\right]^{\#}$ and this is equal to $\left(\alpha_{\#} x * \beta_{\#} y\right)^{\#}$ by Lemma 6.3 .

By the same argument we have the following.

Proposition 6.5. If $\mu, \mu^{\prime}: T(H) \otimes T(H) \rightarrow T(H)$ are given by two uniform families $F, F^{\prime}$ then there is a uniform homotopy $G: F \simeq F^{\prime}$ and $G$ gives a homotopy $g: \mu \simeq \mu^{\prime}$ by

$$
g\left(S_{a}(n, x), S_{b}(m, y)\right)=(x * y)^{\#} G_{a b}(n, m)
$$




\section{Associativity of $\mu$ FOR $K M$}

We will now verify algebraically that the multiplication $\mu$ on the cyclic homology of $H$ satisfies the various conditions that we have claimed except for the computation in Theorem 1.1b in the case $H=K$ and Corollary 1.2. For these we must rely on the topological argument and the proof given at the end of the paper that the topological and algebraic definitions of $\mu$ agree.

The first property that we will tackle is the associativity of $\mu$ in the case when $*$ is associative. As in the case of Proposition 6.5 (the uniqueness of $\mu$ up to homotopy), a homotopy $\mu(\mu \times 1) \simeq \mu(1 \times \mu)$ in the case of a bialgebra $H$ results from a uniform homotopy in the case $H=K M$ which in turn comes from a specific homotopy in the case $M=\{1\}$. The key point therefore is to show that the chain maps $\mu(\mu \times 1), \mu(1 \times \mu): T(K M) \otimes T(K M) \otimes T(K M) \rightarrow T(K M)$ are carried by the same acyclic carrier $A$ which is functorial in $M$. In this section we construct the carrier $A$ and use it to show that $\mu(\mu \times 1), \mu(1 \times \mu)$ are uniformly homotopic. We assume that the bimultiplicative pairing $*: M \times M \rightarrow M$ is associative.

Let $\left(S_{a}(n, x), S_{b}(m, y), S_{c}(l, z)\right)$ be an object of the basis category $\mathcal{B}\left(M^{\Lambda}\right) \times$ $\mathcal{B}\left(M^{\Lambda}\right) \times \mathcal{B}\left(M^{\Lambda}\right)$ of $T(K M) \otimes T(K M) \otimes T(K M)$. We define $x * y * z$ to be the element of $M^{(n+1)(m+1)(l+1)}$ with $(i, j, k)$-coordinate $x_{i} * y_{j} * z_{k}$ and we define the augmented complex $A(x * y * z)$ to be the acyclic complex $E(Z[n, m, l])$ where $Z[n, m, l]=Z[n] \times$ $Z[m] \times Z[l]$ together with the augmented chain map $(x * y * z)^{\#}: E(Z[n, m, l]) \rightarrow$ $T(K M)$ induced by the map of cyclic sets $(x * y * z)^{\#}: X(Z[n, m, l]) \rightarrow M^{\Lambda}$ which sends $\left(\phi: Z[n, m, l] \rightarrow Z[p] \in X_{p} Z[n, m, l]\right)$ to $\phi_{\#}(x * y * z)=\left(w_{0}, \ldots, w_{q}\right) \in M^{q+1}$ where each $w_{t}$ is the product $w_{t}=\prod\left\{x_{i} * y_{j} * z_{k} \mid \bar{\phi}(i, j, k)=t\right\}$. As before we get an acyclic carrier $A$.

Theorem 7.1. The acyclic carrier $A$ carries both $\mu(\mu \times 1)$ and $\mu(1 \times \mu)$. Therefore $\mu$ is homotopy associative on $T(K M)$.

Remark. This is a special case of Theorem 13.1 below.

Proof. We will show that $\mu(\mu \times 1)$ is carried by $A$. The other case is similar.

Recall that $\mu\left(S_{a}(n, x), S_{b}(m, y)\right)=(x * y)^{\#} F_{a b}(n, m)$ where $F_{a b} \in E_{k}(Z[n, m])$, $k=n+m+2(a+b)$. Let

$$
F_{a b}(n, m)=\sum_{\phi} r_{\phi S_{d}(k-2 d, \phi)}
$$

be an expression for $F_{a b}(n, m)$ as a linear combination of generators of $E_{k}(Z[n, m])$. Then

$$
(x * y)^{\#} F_{a b}(n, m)=\sum_{\phi} r_{\phi} S_{d}\left(k-2 d, \phi_{\#}(x * y)\right)
$$

and

$$
\begin{aligned}
\mu\left(\mu\left(S_{a}(n, x), S_{b}(m, y)\right), S_{c}(l, z)\right) & =\mu\left((x * y)^{\#} F_{a b}(n, m), S_{c}(l, z)\right) \\
& =\sum_{\phi} r_{\phi}\left(\phi_{\#}(x * y) * z\right)^{\#} F_{d c}(k-2 d, l) .
\end{aligned}
$$

But $\left(\phi_{\#}(x * y) * z\right)^{\#}=(x * y * z)^{\#}(\phi \times 1)^{*}$ by Lemma 4.1 so we have

$$
\mu(\mu \times 1)\left(S_{a}(n, x), S_{b}(m, y), S_{c}(l, z)\right)=(x * y * z)^{\#} \sum_{\phi} r_{\phi}(\phi \times 1)^{*} F_{d c}(k-2 d, l)
$$


where $G_{a b c}(n, m, l)=\sum_{\phi} r_{\phi}(\phi \times 1)^{*} F_{d c}(k-2 d, l) \in E(Z[n, m, l])$ depends only on $a, b, c, n, m, l$. This is a uniform formula for $\mu(\mu \times 1)$ lifting it to the carrier $A$. We need only check the boundary conditions:

$$
\begin{aligned}
& \varepsilon G_{000}(0,0,0)=1, \\
& d G_{a b c}(n, m, l)= \sum_{\alpha \in W(a, n)} \operatorname{sgn}(\alpha)(\alpha \times 1 \times 1)^{*} G_{* c}(*, m, l) \\
&+(-1)^{n} \sum_{\beta \in W(b, m)} \operatorname{sgn}(\beta)(1 \times \beta \times 1)^{*} G_{a^{*} c}(n, *, l) \\
&+(-1)^{n+m} \sum_{\gamma \in W(c, l)} \operatorname{sgn}(\gamma)(1 \times 1 \times \gamma)^{*} G_{a b^{*}}(n, m, *) .
\end{aligned}
$$

The verification of $(3)$ is easy. Since $F_{00}(0,0)=S_{0}\left(0, p_{1}\right), G_{000}(0,0,0)=$ $\left(p_{1} \times 1\right)^{*} S_{0}\left(0, p_{1}\right)$ has augmentation 1 . To verify (4) we begin by taking the boundary of (1):

$$
d F_{a b}(n, m)=\sum_{\phi} r_{\phi} d S_{d}(k-2 d, \phi)=\sum_{\phi} r_{\phi} \sum_{\psi \in W(d, k-2 d)} \operatorname{sgn}(\psi) S_{q}(p, \psi \phi) .
$$

For fixed $c, l$ let the operator $L: E(Z[n, m]) \rightarrow E(Z[n, m, l])$ be defined by

$$
L\left(S_{q}(p, \lambda)\right)=(\lambda \times 1)^{*} F_{q c}(p, l) .
$$

( $L$ is not a chain map. It is a graded homomorphism of degree $l+2 c$.) Then $G_{a b c}(n, m, l)=L F_{a b}(n, m)$ so $L$ transforms (5) and $F_{a b}(n, m)$ into the following equation.

$$
\begin{aligned}
\sum_{\phi} r_{\phi} & \sum_{\psi \in W(d, k-2 d)} \operatorname{sgn}(\psi)(\psi \phi \times 1)^{*} F_{q c}(p, l) \\
= & \sum_{\alpha \in W(a, n)} \operatorname{sgn}(\alpha)(\alpha \times 1 \times 1)^{*} G_{* b c}(*, m, l) \\
& +(-1)^{n} \sum_{\beta \in W(b, m)} \operatorname{sgn}(\beta)(1 \times \beta \times 1)^{*} G_{a^{*} c}(n, *, l) .
\end{aligned}
$$

Compare this with

$$
\begin{aligned}
& d G_{a b c}(n, m, l)=\sum_{\phi} r_{\phi}(\phi \times 1)^{*} d F_{d c}(k-2 d, l) \\
& =\sum_{\phi} r_{\phi}(\phi \times 1)^{*}\left[\sum_{\psi \in W(d, k-2 d)} \operatorname{sgn}(\psi)(\psi \times 1)^{*} F_{q c}(p, l)\right. \\
& \left.\quad+(-1)^{k} \sum_{\gamma \in W(c, l)} \operatorname{sgn}(\gamma)(1 \times \gamma)^{*} F_{d^{*}}(k-2 d, *)\right] \\
& =\sum_{\phi} r_{\phi} \sum_{\psi \in W(d, k-2 d)} \operatorname{sgn}(\psi)(\psi \phi \times 1)^{*} F_{q c}(p, l) \\
& \quad+(-1)^{k} \sum_{\phi} r_{\phi} \sum_{\gamma \in W(c, l)} \operatorname{sgn}(\gamma)(\phi \times \gamma)^{*} F_{d^{*}}(k-2 d, *) .
\end{aligned}
$$


By (6) the $\sum_{\phi} \sum_{\psi}$ term in (7) agrees with the sum of the $\sum_{\alpha}$ and $\sum_{\beta}$ terms in (4). The following computation shows that the $\sum_{\phi} \sum_{\gamma}$ term in (7) agrees with the $\sum_{\gamma}$ term in (4).

$$
G_{a b ?}(n, m, ? ?)=\sum_{\phi} r_{\phi}(\phi \times 1)^{*} d F_{d ?}(k-2 d, ? ?)
$$

so

$$
\begin{aligned}
& (-1)^{n+m} \sum_{\gamma \in W(c, l)} \operatorname{sgn}(\gamma)(1 \times 1 \times \gamma)^{*} G_{a b ?}(n, m, ? ?) \\
& =(-1)^{k} \sum_{\gamma} \operatorname{sgn}(\gamma)(1 \times 1 \times \gamma)^{*}(\phi \times 1)^{*} F_{d ?}(k-2 d, ? ?) \\
& =(-1)^{k} \sum_{\gamma} \operatorname{sgn}(\gamma)(\phi \times \gamma)^{*} F_{d ?}(k-2 d, ? ?) . \quad \square
\end{aligned}
$$

\section{Associativity of $\mu$ FOR Bialgebras $H$}

We now consider the case when $H$ is a commutative, cocommutative $K$-bialgebra with associative superproduct $*$.

Lemma 8.1. If $x=\left(x^{0}, \ldots, x^{n}\right), y=\left(y^{0}, \ldots, y^{n}\right), z=\left(z^{0}, \ldots, z^{l}\right)$ then $(x * y) * z=$ $x *(y * z)$ as elements of $H^{\otimes(n+1)(m+1)(l+1)}$.

Proof. This is an easy computation:

$$
\begin{array}{rlr}
(x * y) * z & =\sum\left(\left(x_{(j)}^{i} * y_{(i)}^{j}\right)_{(k)} * z_{(i)(j)}^{k}\right) & \text { (by definition) } \\
& =\sum\left(\left(x_{(j)(k)}^{i} * y_{(i)(k)}^{j}\right) * z_{(i, j)}^{k}\right) & \text { (by Def. 6.1.1) } \\
& =\sum\left(x_{(j)(k)}^{i} *\left(y_{(i)(k)}^{j} * z_{(i)(j)}^{k}\right)\right) & \text { (by associativity of } \mu) \\
& =\sum\left(x_{(j)(k)}^{i} *\left(y_{(k)(i)}^{j} * z_{(j)(i)}^{k}\right)\right) & \text { by cocommutativity of } \Delta) \\
& =\sum\left(x_{(j)(k)}^{i} *\left(y_{(k)}^{j} * z_{(j)}^{k}\right)_{(i)}\right) & \text { (by Def. 6.1.1) } \\
& =x *(y * z) .
\end{array}
$$

Theorem 8.2. $\mu: T(H) \otimes T(H) \rightarrow T(H)$ is homotopy associative.

Proof. We recall that $\mu$ is given by $\mu\left(S_{a}(n, x), S_{b}(m, y)\right)=(x * y)^{\#} F_{a b}(n, m)$. Thus

$$
\begin{aligned}
\mu\left(\mu\left(S_{a}(n, x), S_{b}(m, y)\right), S_{c}(l, z)\right) & =\mu\left((x * y)^{\#} F_{a b}(n, m), S_{c}(l, z)\right) \\
& =\mu\left(\sum_{\phi} r_{\phi}(x * y)^{\#} S_{d}(k-2 d, \phi), S_{c}(l, z)\right) \\
& =\mu\left(\sum_{\phi} r_{\phi} S_{d}\left(k-2 d, \phi_{\#}(x * y)\right), S_{c}(l, z)\right) \\
& =\sum_{\phi} r_{\phi}\left(\phi_{\#}(x * y) * z\right)^{\#} F_{d c}(k-2 d, l) \\
& =((x * y) * z)^{\#} \sum_{\phi} r_{\phi}(\phi \times 1)^{*} F_{d c}(k-2 d, l) \\
& =((x * y) * z)^{\#} G_{a b c}(n, m, l)
\end{aligned}
$$


where $G_{a b c}(n, m, l)$ is a uniform family for $\mu(\mu \times 1)$. Similarly

$$
\mu\left(S_{a}(n, x), \mu\left(S_{b}(m, y), S_{c}(l, z)\right)\right)=(x *(y * z))^{\#} G_{a b c}^{\prime}(n, m, l)
$$

where $G_{a b c}^{\prime}(n, m, l)$ is a uniform family for $\mu(1 \times \mu)$. By Theorem 7.1 the uniform families $G, G^{\prime}$ are equivalent to chain maps $g, g^{\prime}: T(K) \otimes T(K) \otimes T(K) \rightarrow T(K)$ carried by the carrier $A$. Therefore any homotopy $h: g \simeq g^{\prime}$ carried by $A$ is equivalent to a uniform homotopy $G \simeq G^{\prime}$. Since $(x * y) * z=x *(y * z)$ by Lemma 8.1 we are done.

\section{Commutativity and unit For $\mu$}

By an easy variation of the associativity argument we can prove the following. This general pattern of argument is later formalized in sections 12 and 13 although the commutativity of $\mu$ is not a special case of Theorem 13.1.

Theorem 9.1. If $*$ is commutative then $\mu$ is homotopy skew commutative on $H C_{*}(H)$.

Proof. Let $w: T(H) \otimes T(H) \rightarrow T(H) \otimes T(H)$ and $\omega: Z[n, m] \rightarrow Z[m, n]$ be the switching maps $w(x, y)=(-1)^{\operatorname{deg} x \operatorname{deg} y}(y, x)$ and $\bar{\omega}(a, b, k)=(b, a, k)$. Then we want to show that $\mu, \mu \circ w$ are uniformly homotopic for $H=K M$. First we note that $\mu\left(S_{a}(n, x), S_{b}(m, y)\right)=(x * y)^{\#} F_{a b}(n, m)$ and

$$
\mu w\left(S_{a}(n, x), S_{b}(m, y)\right)=(-1)^{n m}\left(S_{b}(m, y), S_{a}(n, x)\right)=(-1)^{n m}(y * x)^{\#} F_{b a}(m, n) .
$$

But $F_{a b}^{\prime}(n, m)=(-1)^{n m} \omega^{*} F_{b a}(m, n)$ is a uniform formula for $\mu$ and $(y * x)^{\#} \omega^{*}=$ $\left(\omega_{\#}(y * x)\right)^{\#}=(x * y)^{\#}$. Consequently a uniform homotopy $F \simeq F^{\prime}$ gives a homotopy $\mu \simeq \mu \circ w$.

In the bialgebra case we need to note that $\omega_{\#}(y * x)=x * y$ in $H^{\otimes(n+1)(m+1)}$.

Theorem 9.2. Suppose that $e \in M$ acts as a two-sided unit for $*$. Then $S_{0}(0, e) \in$ $T_{0}(K M)$ acts as a homotopy unit for $\mu .\left(S_{0}(0, e)\right.$ is a strict two-sided unit for $\mu$ if $F$ is chosen as in Proposition 5.3).

Proof. We may assume that $F$ is as in Proposition 5.3 since it is unique up to homotopy. Then:

$$
\begin{aligned}
\mu\left(S_{a}(n, x), S_{0}(0, e)\right) & =(x * e)^{\#} F_{a 0}(n, 0)=x^{\#} S_{a}\left(n, p_{1}\right)=S_{a}(n, x), \\
\mu\left(S_{0}(0, e), S_{b}(m, y)\right) & =(e * y)^{\#} F_{0 b}(0, m)=y^{\#} S_{b}\left(m, p_{2}\right)=S_{b}(m, y) .
\end{aligned}
$$

Theorem 9.3. Suppose that $H$ is a commutative, cocommutative bialgebra and $*$ is a superproduct with unit $e$. Then $S_{0}(0, e)$ is a homotopy unit for $\mu$.

Proof. Choose $F$ as in Proposition 5.3. Then $S_{0}(0, e)$ is a strict two-sided unit for $\mu$ by the calculation above and the fact that $x * e=e * x=x$ for all $x \in H^{\otimes(n+1)}$ which in turn follows from the assumption $\Delta(e)=e \otimes e$.

\section{THE POWER OPERATION $\lambda^{k}$}

If $P$ is a cyclically ordered set and $k \geq 1$ then let $\lambda^{k} P$ be the set $P$ with a new partial ordering $\leq_{k}$ on $\widetilde{P}=P \times \mathbb{Z}$ given by $(x, n) \leq_{k}(y, m)$ if and only if $(x, 0) \leq(y,[(m-n) / k])$ where [ ] denotes the greatest integer function. Since the new partial ordering $\leq_{k}$ on $\widetilde{P}$ is weaker than the original partial ordering the identity map on $\widetilde{P}$ gives a morphism $\lambda^{k} P \rightarrow P$. However this morphism is not 
natural. The reader can check that for $P=Z[n]$ the definition of $\lambda^{k} Z[n]$ agrees with the original one given in Definition 3.3.

If $\phi: P \rightarrow Q$ is a morphism of cyclically ordered sets then let $\lambda^{k} \phi: \lambda^{k} P \rightarrow \lambda^{k} Q$ be given by $\lambda^{k} \tilde{\phi} \sigma^{n}(x)=\sigma^{n} J_{k} \tilde{\phi}(x)$ where $J_{k}$ is the operation $J_{k}(y, m)=(y, k m)$ and $\tilde{\phi}: \widetilde{P} \rightarrow \widetilde{Q}$ is a representative of $\phi$.

Proposition 10.1. (a) $(x, n)<(y, m)$ in $\widetilde{P}$ if and only if $(x, k n)<(y, k m)$ in $\lambda^{k} \widetilde{P}$, i.e., $J_{k}: \widetilde{P} \rightarrow \lambda^{k} \widetilde{P}$ is a poset embedding.

(b) $J_{k} \sigma=\sigma^{k} J_{k}$ (in particular $J_{k}$ is not a morphism of cyclically ordered sets).

(c) $\lambda^{k}$ is a functor from the category of cyclically ordered sets to itself.

(d) If $\phi: P \rightarrow Q$ is a morphism of cyclically ordered sets, then the following diagram commutes.

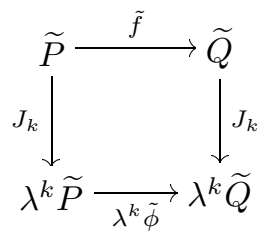

Proof. We will verify that $\lambda^{k} \tilde{\phi}: \lambda^{k} \widetilde{P} \rightarrow \lambda^{k} \widetilde{Q}$ is order preserving. The rest is obvious.

Suppose that $(x, n) \leq(y, m)$ in $\lambda^{k} \widetilde{P}$. Then $(x, 0) \leq(y, a)$ in $\widetilde{P}$ where $a k \leq m-n$. Thus $\tilde{\phi}(x) \leq \sigma^{a} \tilde{\phi}(y)$ in $\widetilde{Q}$ and $J_{k} \tilde{\phi}(x) \leq \sigma^{a k} J_{k} \tilde{\phi}(y) \leq \sigma^{m-n} J_{k} \tilde{\phi}(y)$ in $\lambda^{k} \widetilde{Q}$. But then $\lambda^{k} \tilde{\phi}(x, n)=\sigma^{n} J_{k} \tilde{\phi}(x) \leq \sigma^{m} J_{k} \tilde{\phi}(y)=\lambda^{k} \tilde{\phi}(y, m)$ in $\lambda^{k} \widetilde{Q}$.

Proposition 10.2. For any two cyclically ordered sets $P, Q$ there is a natural isomorphism

$$
\lambda^{k}(P \times Q) \cong \lambda^{k} P \times \lambda^{k} Q .
$$

Proof. We first claim that the "identity map" gives an isomorphism $\lambda^{k}(P \times Q) \cong$ $\lambda^{k} P \times \lambda^{k} Q$. To see this note that the following are equivalent.

(a) $(a, b, n) \leq(x, y, m)$ in $\lambda^{k}(P \times Q)$.

(b) $(a, b, 0) \leq(x, y,[(m-n) / k])$ in $P \times Q$.

(c) $\exists i$ s.t. $(a, 0) \leq(x, i)$ in $P$ and $(b, 0) \leq(y,[(m-n) / k]-i)$ in $Q$.

(d) $\exists i$ s.t. $(a, 0) \leq(x, k i)$ in $\lambda^{k} P$ and $(b, n) \leq(y, m-k i)$ in $Q$.

(e) $(a, b, n) \leq(x, y, m)$ in $\lambda^{k} P \times \lambda^{k} Q$.

Now suppose that $\phi: P \rightarrow S, \psi: Q \rightarrow T$ are morphisms. Then

$$
\lambda^{k}(\widetilde{\phi \times \psi})(x, y, 0)=J_{k}(\widetilde{\phi \times \psi})(x, t, 0)=(s, t, k(a+b))
$$

where $\tilde{\phi}(x, 0)=(s, a)$ and $\tilde{\psi}(y, 0)=(t, b)$. But then $\lambda^{k} \tilde{\phi}(x, 0)=(s, k a)$ and $\lambda^{k} \tilde{\psi}(y, 0)=(t, k b)$ so

$$
\left(\lambda^{k} \tilde{\phi} \times \lambda^{k} \tilde{\psi}\right)(x, y, 0)=(s, t, k a+k b)=\lambda^{k}(\widetilde{\phi \times \psi})(x, y, 0) .
$$

\section{The Power operations on $H C_{*}(A)$}

We will give an acyclic carrier for the power operations $\Lambda^{k}=1 / k \Psi^{k}$ on $T(A)$ for any commutative $K$-algebra $A$. This will enable us to show that $\Psi^{k}$ commutes with $\mu$ up to uniform homotopy. As before we will not need an explicit formula for $\Psi^{k}$ except to show that our operations agree with those of $[\mathrm{L}]$ and $[\mathrm{FT}]$. 
As always we begin with the case $A=K M$ where $M$ is a commutative monoid. For each $k \geq 1$ let $\Lambda^{k}: T(K M) \rightarrow T(K M)$ be any chain map carried by the acyclic carrier $C$ given by $C\left(S_{a}(n, x)\right)=E\left(\lambda^{k} Z[n]\right)$ together with the chain map $x^{\#}$ : $E\left(\lambda^{k} Z[n]\right) \rightarrow T(K M)$ induced by the morphism of cyclic sets $x^{\#}: X\left(\lambda^{k} Z[n]\right) \rightarrow$ $M^{\Lambda}$ given by $x^{\#}(\phi)=\phi_{\#}=\left(z_{0}, \ldots, z_{m}\right)$ where $z_{j}=\prod\left\{x_{i} \mid \bar{\phi}(i)=j\right\}$. A morphism $S_{a}(n, x) \rightarrow S_{b}\left(m, \alpha_{\#} x\right)$ given by $\alpha: Z[n] \rightarrow Z[m]$ induces a chain map $\left(\lambda^{k} \alpha\right)^{*}$ : $E\left(\lambda^{k} Z[m]\right) \rightarrow E\left(\lambda^{k} Z[n]\right)$.

To verify that this is a carrier we note that $\left(\lambda^{k} \alpha\right)^{*}\left(\lambda^{k} \beta\right)^{*}=\lambda^{k}(\beta \alpha)^{*}$ and $x^{\#}\left(\lambda^{k} \alpha\right)^{*}=\lambda^{k} \alpha_{\#}(x)^{\#}=\left(\alpha_{\#} x\right)^{\#}$ since $\alpha, \lambda^{k} \alpha$ give the same map of underlying sets $Z[n] \rightarrow Z[m]$.

As before we get a uniform formula for $\Psi^{k}$ :

$$
\Lambda^{k}\left(S_{a}(n, x)\right)=x^{\#} L_{a}^{k}(n)
$$

where $L_{a}^{k}(n) \in E_{n+2 a}\left(\lambda^{k} Z[n]\right)$ is a family of elements depending only on $a, n, k$.

Proposition 11.1. The uniform family of elements $L_{a}^{k}(n)$ is characterized (up to uniform homotopy) by the following properties.

(a) $\varepsilon L_{0}^{k}(0)=1$,

(b)

$$
\begin{aligned}
\partial L_{a}^{k}(n)=\sum_{i=0}^{n}\left[(-1)^{i}\left(\lambda^{k} \partial_{i}\right)^{*} L_{a}^{k}(n-1)\right. & +(-1)^{n i}\left(\lambda^{k} s t^{i}\right)^{*} L_{a-1}^{k}(n+1) \\
& \left.+(-1)^{n i+n}\left(\lambda^{k} t s t^{i}\right)^{*} L_{a-1}^{k}(n+1)\right] .
\end{aligned}
$$

With this characterization we can compare $L_{a}^{k}(n)$ with the power operations $\Psi^{k}$ as described in [FT].

Theorem 11.2. An explicit formula for $L_{a}^{k}(n) \in E_{n+2 a}\left(\lambda^{k} Z[n]\right)$ modulo generacies is given as follows.

$$
L_{a}^{k}(n)=k^{a} \sum_{I} \operatorname{sgn}\left(\phi_{I}\right) S_{a}\left(n, \phi_{I}\right)+\text { degenerate terms }
$$

where $I=\left(I_{1}, \ldots, I_{k}\right)$ runs over all partitions of the set $\{1,2, \ldots, n\}$ and $\phi_{I}$ : $\lambda^{k} Z[n] \rightarrow Z[n]$ is the morphism given by $\tilde{\phi}_{I}(0)=0$ and $\tilde{\phi}_{I}(i, 0)=\left(a_{i}, \pi(i)\right)$ for $i=1, \ldots, n$ where $a_{1}, a_{2}, \ldots, a_{n}$ are the elements of $I_{1}$ in increasing order followed by the elements of $I_{2}$ in increasing order etc. and $\pi(i)=j$ if $a_{i} \in I_{j}$.

Proof. This is a computation which can be broken into two parts.

$$
\begin{gathered}
\sum_{i=0}^{n}(-1)^{i} \partial_{i} \sum_{I} \operatorname{sgn}\left(\bar{\phi}_{I}\right) S_{a}\left(n, \phi_{I}\right)=\sum_{j=0}^{n}(-1)^{j}\left(\lambda^{k} \partial_{j}\right)^{*} \sum_{J} \operatorname{sgn}\left(\bar{\phi}_{J}\right) S_{a}\left(n-1, \phi_{J}\right), \\
k \sum_{i=0}^{n}(-1)^{n i} s^{i} \sum_{I} \operatorname{sgn}\left(\bar{\phi}_{I}\right) S_{a}\left(n, \phi_{I}\right) \\
=\sum_{j=0}^{n}(-1)^{n j}\left(\lambda^{k} s t^{j}\right)^{*} \sum_{L} \operatorname{sgn}\left(\bar{\phi}_{L}\right) S_{a-1}\left(n+1, \phi_{L}\right) .
\end{gathered}
$$


Comparison of the remaining terms shows that they are not equal:

$$
\begin{aligned}
& k \sum_{i=0}^{n}(-1)^{n i+n} t x t^{i} \sum_{I} \operatorname{sgn}\left(\bar{\phi}_{I}\right) S_{a}\left(n, \phi_{I}\right) \\
& \quad \neq \sum_{j=0}^{n}(-1)^{n j+n}\left(\lambda^{k} t s t^{j}\right)^{*} \sum_{L} \operatorname{sgn}\left(\bar{\phi}_{L}\right) S_{a-1}\left(n+1, \phi_{L}\right) .
\end{aligned}
$$

However the expressions in (3) are all degenerate so $L_{a}^{k}(n)=k^{a} \sum_{I} \pm S_{a}\left(n, \phi_{I}\right)$ satisfies 11.1.b up to degeneracies. (Since this formula for $L_{a}^{k}(n)$ sends degeneracies to degeneracies the degeneracies can be factored out.)

To verify equation (1) we rewrite it in the following form

$$
\sum_{i=0}^{n} \sum_{I}(-1)^{i} \operatorname{sgn}\left(\bar{\phi}_{I}\right) S_{a}\left(n-1, \partial_{i} \phi_{I}\right)=\sum_{j=0}^{n} \sum_{J}(-1)^{j} \operatorname{sgn}\left(\bar{\phi}_{J}\right) S_{a}\left(n-1, \phi_{J} \circ \partial_{j}\right) .
$$

If $0<i<n$ and $i, i+1$ belong to different parts of the partition $I$, then $\partial_{i} \phi_{i}=\partial_{i} \phi_{I}$, where $I^{\prime}$ is obtained from $I$ by interchanging $i, i+1$. These terms occur with opposite sign on the left side of (4) and so they cancel. The terms with $\partial_{0} \phi_{I}$ where $1 \in I_{1}$ and $\partial_{n} \phi_{I}$ where $n \in I_{k}$ also cancel. The remaining terms are equal to corresponding terms on the right hand side of (4).

Equation (2) can be rewritten as:

$$
\begin{aligned}
k \sum_{i=0}^{n} & \sum_{I}(-1)^{n i} \operatorname{sgn}\left(\bar{\phi}_{I}\right) S_{a}\left(n, s t^{i} \phi_{I}\right) \\
& =\sum_{j=0}^{n} \sum_{L}(-1)^{n j} \operatorname{sgn}\left(\bar{\phi}_{L}\right) S_{a-1}\left(n+1, \phi_{L} \circ \lambda^{k} s t^{j}\right) .
\end{aligned}
$$

Since $s t^{i} \phi_{I}$ sends 0 to $i+1$ one can see that the morphisms $s t^{i} \phi_{L}$ are all different and that each of them occurs exactly $k$ times (with the same sign) as a morphism of the form $\phi_{L} \circ \lambda^{k} s t^{j}$.

Corollary 11.3. $k \Lambda^{k}=\Psi^{k}=(-1)^{k} k \lambda^{k}$.

Corollary 11.4. Let $A$ be a commutative $K$-algebra. Then the power operation $\Psi^{k}=k \Lambda^{k}$ on $T(A)$ is given by $\Psi^{k} S_{a}(n, x)=k x^{\#} L_{a}^{k}(n)$.

\section{General operations on $H C_{*}(H)$}

We want to show that the power operations $\Lambda^{k}$ commute with the multiplication $\mu$. This will be a formal consequence of the isomorphism $\lambda^{k} P \times \lambda^{k} Q \cong \lambda^{k}(P \times Q)$ in the category $\mathcal{C}$ os of finite nonempty cyclically ordered sets.

In this section we will show how any functor $f: \mathcal{C}^{n} s^{n} \rightarrow \mathcal{C}$ os gives rise to an operation on $H C_{*}(K M)$ for any " $f$-monoid" $M$ and on $H C_{*}(H)$ for any " $f$ algebra" $H$.

Definition 12.1. Let $f: \mathcal{C} \cos \times \cdots \times \operatorname{Cos} \rightarrow \mathcal{C}$ os be a functor of $n$ variables. We say that $f$ acts on the commutative monoid $M$ (and we call $M$ an $f$-monoid) provided that for any $n$ objects $P_{1}, P_{2}, \ldots, P_{n}$ of $\mathcal{C}$ os there is an $n$-multiplicative map (i.e. multiplicative in each variable)

$$
f_{*}: M^{P_{1}} \times \cdots \times M^{P_{n}} \rightarrow M^{f\left(P_{1}, \ldots, P_{n}\right)}
$$


so that for any $n$ morphisms $\phi^{i}: P_{i} \rightarrow Q_{i}$ the following diagram commutes.

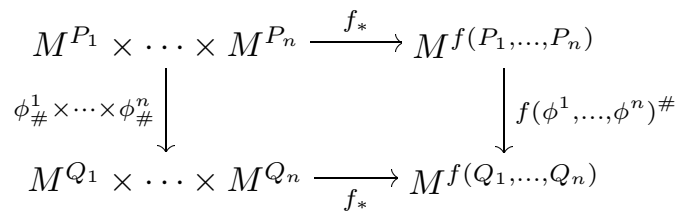

i.e., $f\left(\phi^{1}, \ldots, \phi^{n}\right)_{\#} f_{*}\left(x^{1}, \ldots, x^{n}\right)=f_{*}\left(\phi_{\#}^{1} x^{1}, \ldots, \phi_{\#}^{n} x^{n}\right)$. Here $\phi_{\#}: M^{P} \rightarrow M^{Q}$ is given by $\phi_{\#}(x)=y$ where $y(j)=\prod\{x(i) \mid \bar{\phi}(i)=j\}$.

Note that there is a unique action of any functor $f$ on the trivial monoid $\{1\}$.

Suppose that $f, g: \mathcal{C}_{o s}{ }^{n} \rightarrow \mathcal{C}$ os are two functors acting on the monoids $M, N$ respectively. Then a morphism $(M, f) \rightarrow(N, g)$ of monoid-functor pairs consists of a homomorphism of monoids $h: M \rightarrow N$ and a natural transformation $\eta: f \rightarrow g$ making the following diagram commute for all $P_{1}, \ldots, P_{n}$ in $\mathcal{C}$ os.

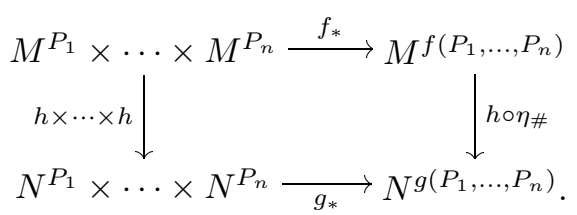

If $\eta: f \rightarrow f$ is the identity transformation we call $h: M \rightarrow N$ an $f$-morphism. Note that for any $f$-monoid $M$ there are unique $f$-morphisms $M \rightarrow\{1\}$ and $\{1\} \rightarrow M$.

Examples 12.2. (a) $f=\lambda^{k}, \lambda_{*}^{i}=$ "identity" : $M^{P} \rightarrow M^{\lambda^{k} P}$, in particular any commutative monoid has the natural "trivial" structure as a $\lambda^{k}$-monoid.

(b) $\mu(P, Q)=P \times Q, \mu_{*}: M^{P} \times M^{Q} \rightarrow M^{P \times Q}$ given by $\mu_{*}(x, y)=x * y,(x *$ $y)(i, j)=x(i) * y(j)$ where $*: M \times M \rightarrow M$ is a bimultiplicative pairing so that $1 * x=x * 1=1$ for all $x \in M$. (The commutativity of (1) in this case is given by Lemma 4.1.)

By replacing products with tensor products these definitions can be extended to $K$-algebras.

Definition 12.3. We say that the functor $f: \mathcal{C}_{\text {os }}{ }^{n} \rightarrow \mathcal{C}$ os acts on a commutative $K$-algebra $H$ and we call $H$ an $f$-algebra if for any $P_{1}, \ldots, P_{n} \in \mathcal{C}$ os there is a $K$-linear map $f_{*}: H^{\otimes P_{1}} \otimes \cdots \otimes H^{\otimes P_{n}} \rightarrow H^{\otimes f\left(P_{1}, \ldots, P_{n}\right)}$ so that for any $n$ morphisms $\phi^{i}: P_{i} \rightarrow Q_{i}$ the following "tensor analogue" of diagram (1) commutes.

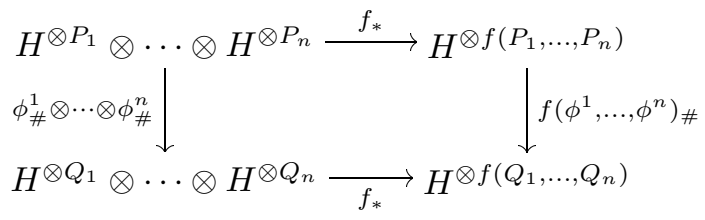

Here $\phi_{\#}: H^{\otimes P} \rightarrow H^{\otimes Q}$ is given on generators $x \in H^{P}$ by $\phi_{\#}(x)=y \in H^{Q}, y(j)=$ $\prod\{x(i) \mid \bar{\phi}(i)=j\}$.

Examples 12.4. (a) Any commutative $K$-algebra has a natural "trivial" $\lambda^{k}$-action.

(b) Any commutative, cocommutative $K$-algebra with superproduct is a $\mu$ algebra where $\mu$ is the functor $\mu(P, Q)=P \times Q$. (This follows from Lemma 6.3.b.) 
A morphism of algebra-functor pairs $(H, f) \rightarrow\left(H^{\prime}, g\right)$ is a $K$-algebra morphism $h: H \rightarrow H^{\prime}$ and a natural transformation $\eta: f \rightarrow g$ making the obvious diagram (the tensor analogue of (2)) commute.

If $f: \mathcal{C o s}^{k} \rightarrow \mathcal{C}$ os is a functor which acts on the commutative monoid $M$ let $A_{f}$ be the acyclic carrier from $T(K M) \otimes \cdots \otimes T(K M)$ to $T(K M)$ given as follows.

(a) If $a_{1}, n_{1}, \ldots, a_{k}, n_{k} \geq 0$ and $x^{i}=\left(x^{i}(0), \ldots, x^{i}\left(n_{i}\right)\right): Z\left[n_{i}\right] \rightarrow M$ are set functions then

$$
A_{f}\left(S_{a_{1}}\left(n_{1}, x^{1}\right), \ldots, S_{a_{k}}\left(n_{k}, x^{k}\right)\right)=E\left(f\left(Z\left[n_{1}\right], \ldots, Z\left[n_{k}\right]\right)\right)
$$

together with the map to $T(K M)=K \mathcal{B}\left(M^{\Lambda}\right)$ induced by the morphism of cyclic sets

$$
f_{*}\left(x^{1}, \ldots, x^{k}\right)^{\#}: X f\left(Z\left[n_{1}\right], \ldots, Z\left[n_{k}\right]\right) \rightarrow M^{\Lambda}
$$

given as before by $f_{*}\left(x^{1}, \ldots, x^{k}\right)^{\#} \phi=\phi_{\#}\left(f_{*}\left(x^{1}, \ldots, x^{k}\right)\right)$. (Note that $f_{*}\left(x^{1}, \ldots, x^{k}\right)$ maps $f\left(Z\left[n_{1}\right], \ldots, Z\left[n_{k}\right]\right)$ into $M$ and $\left.\phi: f\left(Z\left[n_{1}\right], \ldots, Z\left[n_{k}\right]\right) \rightarrow Z[?].\right)$

(b) If $\phi^{i}: Z\left[n_{i}\right] \rightarrow Z\left[m_{i}\right], i=1,2, \ldots, k$, are morphisms then there is an induced map

$$
f\left(\phi^{1}, \ldots, \phi^{k}\right)^{*}: E\left(f\left(Z\left[m_{1}\right], \ldots, Z\left[m_{k}\right]\right)\right) \rightarrow E\left(f\left(Z\left[n_{1}\right], \ldots, Z\left[n_{k}\right]\right)\right) .
$$

In order to show that this defines an acyclic carrier we need to show that the following diagram commutes:

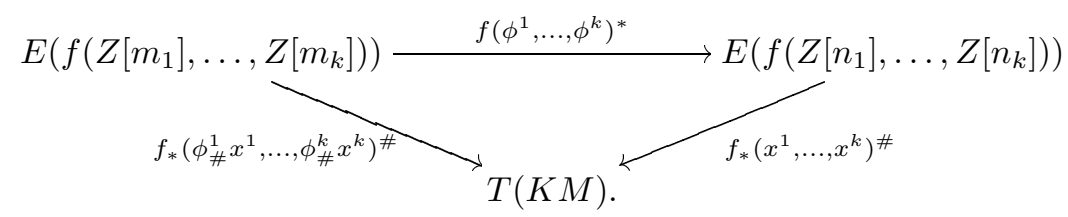

This is a familiar calculation:

$$
\begin{aligned}
f_{*}\left(x^{1}, \ldots, x^{k}\right)^{\#} f\left(\phi^{1}, \ldots, \phi^{k}\right)^{*}(\psi) & =f_{*}\left(x^{1}, \ldots, x^{k}\right)^{\#}\left(\psi \circ f\left(\phi^{1}, \ldots, \phi^{k}\right)\right) \\
& =\psi_{\#} f\left(\phi^{1}, \ldots, \phi^{k}\right)_{\#} f_{*}\left(x^{1}, \ldots, x^{k}\right) \\
& =\psi_{\#} f_{*}\left(\phi_{\#}^{1} x^{1}, \ldots, \phi_{\#}^{k} x^{k}\right) \\
& =f_{*}\left(\phi_{\#}^{1} x^{1}, \ldots, \phi_{\#}^{k} x^{k}\right)^{\#}(\psi) .
\end{aligned}
$$

We also recall that $f\left(Z\left[n_{1}\right], \ldots, Z\left[n_{k}\right]\right)$ is finite and nonempty by assumption.

A uniform formula for a chain map $\theta_{f}$ carried by $A_{f}$ can be given by

$$
\theta_{f}\left(S_{a_{1}}\left(n_{1}, x^{1}\right), \ldots, S_{a_{k}}\left(n_{k}, x^{k}\right)\right)=f_{*}\left(x^{1}, \ldots, x^{k}\right)^{\#} F_{f}\left(a_{1}, n_{1}, \ldots, a_{k}, n_{k}\right)
$$

where $F_{f}\left(a_{1}, n_{1}, \ldots, a_{k}, n_{k}\right) \in E_{p}\left(f\left(Z\left[n_{1}\right], \ldots, Z\left[n_{k}\right]\right)\right)$ with $p=n_{1}+\cdots+n_{k}+$ $2\left(a_{1}+\cdots+a_{k}\right)$ is a family of elements given uniquely up to uniform homotopy by the following conditions.

$$
\varepsilon F_{f}(0,0, \ldots, 0,0)=1
$$


(6)

$$
\begin{aligned}
& \partial F_{f}\left(a_{1}, n_{1}, \ldots, a_{k}, n_{k}\right) \\
& =\sum_{\phi^{1} \in W\left(a_{1}, n_{1}\right)} \operatorname{sgn}\left(\phi^{1}\right) f\left(\phi^{1}, 1, \ldots, 1\right)^{*} F_{f}\left(*, *, a_{2}, n_{2}, \ldots, a_{k}, n_{k}\right) \\
& \quad+(-1)^{n_{1}} \sum_{\phi^{2} \in W\left(a_{2}, n_{2}\right)} \operatorname{sgn}\left(\phi^{2}\right) f\left(1, \phi^{2}, 1, \ldots, 1\right)^{*} F_{f}\left(a_{1}, n_{1}, *, *, a_{3}, n_{3}, \ldots, a_{k}, n_{k}\right) \\
& \quad+\cdots+(-1)^{n_{1}+n_{2}+\cdots+n_{k}} \sum_{\phi^{k} \in W\left(a_{k}, n_{k}\right)} \operatorname{sgn}\left(\phi^{k}\right) \\
& \quad \times f\left(1, \ldots, 1, \phi^{k}\right)^{*} F_{f}\left(a_{1}, n_{1}, \ldots, a_{k-1}, n_{k-1}, *, *\right) .
\end{aligned}
$$

Proposition 12.5. Suppose that $f: \mathcal{C}_{o s}{ }^{k} \rightarrow \mathcal{C}$ os acts on the $K$-algebra $H$. Then (4) above defines a chain map $\theta_{f}: T(H) \otimes k \rightarrow T(H)$ and thus an operation $\theta_{f}: H C_{n_{1}}(H) \otimes \cdots \otimes H C_{n_{k}}(H) \rightarrow H C_{n_{1}+\cdots+n_{k}}(H)$ which is natural with respect to morphisms of $f$-algebras. Furthermore this map in cyclic homology is independent of the choice of the uniform family $F_{f}$.

Proof. Since $f_{*}\left(x^{1}, \ldots, x^{k}\right)$ is multilinear, (4) defines a linear map $\theta_{f}$. Condition (6) shows that $\theta_{f}$ commutes with boundary. We just need to check that $f_{*}\left(x^{1}, \ldots, x^{k}\right)^{\#} f\left(\phi^{1}, \ldots, \phi^{k}\right)^{*}=f_{*}\left(\phi_{\#}^{1} x^{1}, \ldots, \phi_{\#}^{k} x^{k}\right)^{\#}$. But this follows from the commutativity of $(3)$.

\section{COMPOSITION OF UNIFORM FORMULAS}

Let $f: \mathcal{C}_{0 s}{ }^{s} \rightarrow \mathcal{C}$ os be a functor of $s$ variables and let $g^{i}: \mathcal{C}_{0 s}{ }^{t_{i}} \rightarrow \mathcal{C}$ os be $s$ functors. Then we get a composite functor $f g=f \circ\left(g^{1} \times \cdots \times g^{s}\right): \mathcal{C}_{o s}{ }^{T} \rightarrow \mathcal{C} o s$ where $T=t_{1}+\cdots+t_{s}$. We will obtain a uniform family for this composite functor in terms of the uniform family $F_{f}$ for $f$ and uniform families $G_{1}, \ldots, G_{s}$ for $g^{1}, \ldots, g^{s}$.

First we need some notation. For each $i=1, \ldots, s$ we have

$$
G_{i}\left(a_{1}, n_{1}, \ldots, a_{t}, n_{t}\right)=\sum_{I_{i}} r_{I_{i}} S_{b_{I_{1}}}\left(m_{I_{1}}, \psi^{I_{i}}\right)
$$

where $\psi^{I_{i}}: g^{i}\left(Z\left[n_{1}\right], \ldots, Z\left[n_{t}\right]\right) \rightarrow Z\left[m_{I_{i}}\right]$ is a morphism, $\left\{I_{i}\right\}$ is an index set depending on $i,\left(a_{1}, n_{1}, \ldots, a_{t}, n_{t}\right)$ and $t=t_{i}$. We note that $m_{I_{i}}+2 b_{I_{i}}=\sum n_{j}+$ $2 \sum a_{j}$ for each $I_{i}$. Since $G_{i}$ is a uniform family we have

$$
\begin{aligned}
& \partial G_{i}\left(a_{1}, n_{1}, \ldots, a_{t}, n_{t}\right)=\sum_{I_{i}} \sum_{\alpha^{i} \in W\left(b_{I_{i}}, m_{I_{i}}\right)} \operatorname{sgn}\left(\alpha^{i}\right) r_{I_{i}} S_{*}\left(*, \alpha^{i} \psi^{I_{i}}\right) \\
& =\sum_{\beta^{i 1} \in W\left(a_{1}, n_{1}\right)} \operatorname{sgn}\left(\beta^{i 1}\right) g^{i}\left(\beta^{i 1}, 1, \ldots, 1\right)^{*} G_{i}\left(*, *, a_{2}, n_{2}, \ldots, a_{t}, n_{t}\right) \\
& \quad+(-1)^{n_{1}} \sum_{\beta^{i 2} \in W\left(a_{2}, n_{2}\right)} \operatorname{sgn}\left(\beta^{i 2}\right) g^{i}\left(1, \beta^{i 2}, 1, \ldots, 1\right)^{*} G_{i}\left(a_{1}, n_{1}, *, *, a_{3}, n_{3}, \ldots, a_{t}, n_{t}\right) \\
& \quad+\cdots+(-1)^{n_{1}+n_{2}+\cdots+n_{t-1}} \sum_{\beta^{i t} \in W\left(a_{t}, n_{t}\right)} \operatorname{sgn}\left(\beta^{i t}\right) \\
& \quad \times g^{i}\left(1, \ldots, 1, \beta^{i t}\right)^{*} G_{i}\left(a_{1}, n_{1}, \ldots, a_{t-1}, n_{t-1}, *, *\right) .
\end{aligned}
$$


We now construct $F_{f g}$.

$$
\begin{aligned}
& \theta_{f}\left(\theta_{1}, \ldots, \theta_{s}\right) /\left(S_{a_{11}}\left(n_{11}, x^{11}\right), S_{a_{12}}\left(n_{12}, x^{12}\right), \ldots, S_{a_{s t}}\left(n_{s t}, x^{s t}\right)\right) \\
& =\theta_{f}\left(g_{*}^{1}\left(x^{11}, \ldots, x^{1 t}\right)^{\#} G_{1}\left(a_{11}, n_{11}, \ldots, a_{1 t}, n_{1 t}\right), \ldots\right. \\
& \left.g_{*}^{s}\left(x^{s 1}, \ldots, x^{s t}\right)^{\#} G_{s}\left(a_{s 1}, n_{s 1}, \ldots, a_{s t}, n_{s t}\right)\right) .
\end{aligned}
$$

By (1) and the multilinearity of $\theta_{f}$ this is equal to:

$$
\begin{aligned}
\sum_{I_{1}} \cdots \sum_{I_{s}} & r_{I_{1}} \cdots r_{I_{s}} \theta_{f}\left(g_{*}^{1}\left(x^{11}, \ldots, x^{1 t}\right)^{\#} S_{b_{I_{1}}}\left(m_{I_{1}}, \psi^{I_{1}}\right), \ldots,\right. \\
& \left.g_{*}^{s}\left(x^{s 1}, \ldots, x^{s t}\right)^{\#} S_{b_{I_{s}}}\left(m_{I_{s}}, \psi^{I_{s}}\right)\right) \\
= & \sum_{I_{1}} \cdots \sum_{I_{s}} r_{I_{1}} \cdots r_{I_{s}} f_{*}\left(\psi_{\#}^{I_{1}} g_{*}^{1}\left(x^{11}, \ldots, x^{1 t}\right), \ldots, \psi^{I_{s}} g_{*}^{s}\left(x^{s 1}, \ldots, x^{s t}\right)\right)^{\#} \\
& \times F_{f}\left(b_{I_{1}}, m_{I_{1}}, \ldots, b_{I_{s}}, m_{I_{s}}\right) \\
= & \sum_{I_{1}} \cdots \sum_{I_{s}} r_{I_{1}} \cdots r_{I_{s}} f_{*}\left(g_{*}^{1}\left(x^{11}, \ldots, x^{1 t}\right), \ldots, g_{*}^{s}\left(x^{s 1}, \ldots, x^{s t}\right)^{\#}\right) \\
& \quad \times f\left(\psi^{I_{1}}, \ldots, \psi^{I_{s}}\right)^{*} F_{f}\left(b_{I_{1}}, m_{I_{1}}, \ldots, b_{I_{s}}, m_{I_{s}}\right) \\
= & f g_{*}\left(x^{11}, \ldots, x^{1 t}, \ldots, x^{s 1}, \ldots, x^{s t}\right)^{\#} F_{f g}\left(a_{11}, n_{11}, \ldots, a_{s t}, n_{s t}\right)
\end{aligned}
$$

where

$F_{f g}\left(a_{11}, n_{11}, \ldots, a_{s t}, n_{s t}\right) \in E\left(f\left(g^{1}\left(Z\left[n_{11}\right], \ldots, Z\left[n_{1 t}\right]\right), \ldots, g^{s}\left(Z\left[n_{s 1}\right], \ldots, Z\left[n_{s t}\right]\right)\right)\right)$

is given by

$$
\begin{array}{r}
F_{f g}\left(a_{11}, n_{11}, \ldots, a_{s t}, n_{s t}\right)=\sum_{I_{1}} \cdots \sum_{I_{s}} r_{I_{1}} \cdots r_{I_{s}} f\left(\psi^{I_{1}}, \ldots, \psi^{I_{s}}\right)^{*} \\
\times F_{f}\left(b_{I_{1}}, m_{I_{1}}, \ldots, b_{I_{s}}, m_{I_{s}}\right)
\end{array}
$$

where $f\left(\psi^{I_{1}}, \ldots, \psi^{I_{s}}\right): f\left(g^{1}\left(Z\left[n_{11}\right], \ldots, Z\left[n_{1 t}\right]\right), \ldots, g^{s}\left(Z\left[n_{s 1}\right], \ldots, Z\left[n_{s t}\right]\right)\right) \rightarrow$ $f\left(Z\left[m_{I_{1}}\right], \ldots, Z\left[m_{I_{s}}\right]\right)$.

Theorem 13.1. $F_{f g}$ is a uniform family for $f g$.

Proof. Since $\theta_{f}, \theta_{1}, \ldots, \theta_{s}, f g_{*}\left(x^{11}, \ldots, x^{s t}\right)^{\#}$ commute with augmentation we have $\varepsilon F_{f g}(0, \ldots, 0)=1$. Thus we need only verify the following boundary condition.

$$
\begin{aligned}
& \partial F_{f g}\left(a_{11}, n_{11}, \ldots, a_{s t}, n_{s t}\right) \\
& ={ }_{(?)} \sum_{\beta^{11} \in W\left(a_{11}, n_{11}\right)} \operatorname{sgn}\left(\beta^{11}\right) f\left(g^{1}\left(\beta^{11}, 1, \ldots, 1\right), 1, \ldots, 1\right)^{*} \\
& \quad \times F_{f g}\left(*, *, a_{12}, n_{12}, \ldots, a_{s t}, n_{s t}\right) \\
& +(-1)^{n_{11}} \sum_{\beta^{12} \in W\left(a_{12}, n_{12}\right)} \operatorname{sgn}\left(\beta^{12}\right) f\left(g^{1}\left(1, \beta^{12}, 1, \ldots, 1\right), 1, \ldots, 1\right)^{*} \\
& \quad \times F_{f g}\left(a_{11}, n_{11}, *, *, a_{13}, n_{s t}\right)+\cdots
\end{aligned}
$$


where the indices of summation $\beta^{i j}$ are as in equation (2). The actual boundary of $F_{f g}\left(a_{11}, \ldots, n_{s t}\right)$ is obtained from (3):

$(5)$

$$
\begin{gathered}
\partial F_{f g}\left(a_{11}, \ldots, n_{s t}\right)=\sum_{I_{1}} \cdots \sum_{I_{s}} r_{I_{1}} \cdots r_{I_{s}} f\left(\psi^{I_{1}}, \ldots, \psi^{I_{s}}\right)^{*} \partial F_{f}\left(b_{I_{1}}, m_{I_{1}}, \ldots, b_{I_{s}}, m_{I_{s}}\right) \\
=\sum_{I_{1}} \cdots \sum_{I_{s}} r_{I_{1}} \cdots r_{I_{s}} f\left(\psi^{I_{1}}, \ldots, \psi^{I_{s}}\right)^{*} \\
\quad \cdot\left[\sum_{\alpha^{1} \in W\left(b_{I_{1}}, m_{I_{1}}\right)} \operatorname{sgn}\left(\alpha^{1}\right) f\left(\alpha^{1}, 1, \ldots, 1\right)^{*} F_{f}\left(*, *, b_{I_{2}}, \ldots, m_{I_{s}}\right)\right. \\
+(-1)^{m_{I_{1}}} \sum_{\alpha^{2} \in W\left(b_{I_{2}}, m_{I_{2}}\right)} \operatorname{sgn}\left(\alpha^{2}\right) f\left(1, \alpha^{2}, 1, \ldots, 1\right)^{*} \\
\left.\times F_{f}\left(b_{I_{1}}, m_{I_{1}}, *, *, b_{I_{3}}, \ldots, m_{I_{s}}\right)+\cdots\right]
\end{gathered}
$$

where $\alpha^{i}$ is as in (2). Thus it suffices to show that the RHS of (4) is equal to the RHS of (5). We will see that this is a formal consequence of (2).

The terms in (2) can be converted into the corresponding terms in (4), (5) via the graded homomorphisms

$$
\begin{aligned}
V_{i} & : E\left(g^{i}\left(Z\left[n_{i 1}\right], \ldots, Z\left[n_{i t}\right]\right)\right) \\
& \rightarrow E\left(f\left(t^{1}\left(Z\left[n_{11}\right], \ldots, Z\left[n_{1 t}\right]\right), \ldots, g^{s}\left(Z\left[n_{s 1}\right], \ldots, Z\left[n_{s t}\right]\right)\right)\right)
\end{aligned}
$$

given for each $c, k \geq 0$ and $\phi: g^{i}\left(Z\left[n_{i 1}\right], \ldots, Z\left[n_{i t}\right]\right) \rightarrow Z[k]$ by

$$
\begin{aligned}
V_{i} S_{c}(k, \phi) & =\sum_{I_{1}} \cdots \sum_{I_{i-1}} \sum_{I_{i+1}} \cdots \sum_{I_{s}} r_{I_{1}} \cdots r_{I_{i-1}} r_{I_{i+1}} \ldots \\
& r_{I_{s}} f\left(\psi^{I_{1}}, \ldots, \psi^{I_{i-1}}, \phi, \psi^{I_{i+1}}, \ldots, \psi^{I_{s}}\right)^{*} \\
\times & F_{f}\left(b_{I_{1}}, m_{I_{1}}, \ldots, b_{I_{i-1}}, m_{I_{i-1}}, c, k, b_{I_{i+1}}, m_{I_{i+1}}, \ldots, b_{I_{s}}, m_{I_{s}}\right) .
\end{aligned}
$$

Although $V_{i}$ is not a chain map $\left(V_{i} \partial \neq \partial V_{i}\right)$ it is natural in the following sense. If $\phi^{j}: Z\left[n_{i j}^{\prime}\right] \rightarrow Z\left[n_{i j}\right]$ are morphisms then

$$
V_{i} \circ g^{i}\left(\phi^{1}, \ldots, \phi^{t}\right)^{*}=f\left(1, \ldots, 1, g^{i}\left(\phi^{1}, \ldots, \phi^{t}\right), 1, \ldots, 1\right)^{*} \circ V_{i}^{\prime}
$$

where $V_{i}^{\prime}$ is the same as $V_{i}$ with each $n_{i j}$ replaced by $n_{i j}^{\prime}$. If we compare the terms in the second expression in (2) with those in the RHS of (5) we see that

(6)

$$
\sum_{i=1}^{s}(-1)^{n_{11}+\cdots+n_{i-1, t}} V_{i}\left(\partial G_{i}\left(a_{i 1}, n_{i 1}, \ldots, a_{i t}, n_{i t}\right)\right)=\partial F_{f g}\left(a_{11}, n_{11}, \ldots, a_{s t}, n_{s t}\right) .
$$

The signs match since $n_{i 1}+\cdots+n_{i t}$ has the same parity as $m_{I_{i}}$. If we apply $V_{i}$ to both sides of (1) we get

$$
\begin{aligned}
V_{i} G_{i}\left(a_{1}, n_{1}, \ldots, a_{t}, n_{t}\right)= & \sum_{I_{1}} \cdots \sum_{I_{s}} r_{I_{1}} \cdots r_{I_{s}} f\left(\psi^{I_{1}}, \ldots, \psi^{I_{s}}\right)^{*} \\
& \times F_{f}\left(b_{I_{1}}, m_{I_{1}}, \ldots, b_{I_{s}}, m_{I_{s}}\right) \\
= & F_{f g}\left(a_{11}, n_{11}, \ldots, a_{s t}, n_{s t}\right) .
\end{aligned}
$$


Now we can use (7) and the naturality of $V_{i}$ to compute $V_{i} \partial G_{i}\left(a_{i 1}, n_{i 1}, \ldots, a_{i t}, n_{i t}\right)$ using the last expression in (2):

$$
\begin{aligned}
& V_{i} \partial G_{i}\left(a_{i 1}, n_{i 1}, \ldots, a_{i t}, n_{i t}\right) \\
& =\sum^{\beta^{i 1} \in W\left(a_{i 1}, n_{i 1}\right)} \operatorname{sgn}\left(\beta^{i 1}\right) f\left(1, \ldots, 1, g^{i}\left(\beta^{i 1}, 1, \ldots, 1\right), 1, \ldots, 1\right)^{*} \\
& \quad \times V_{i} G_{i}\left(*, *, a_{i 2}, n_{i 2}, \ldots, a_{i t}, n_{i t}\right)+\cdots .
\end{aligned}
$$

Comparing this with the RHS of (4) we see that

$$
\begin{aligned}
\operatorname{RHS}(4) & =\sum_{i=1}^{s}(-1)^{n_{11}+\cdots+n_{i-1, t}} V_{i}\left(\partial G_{i}\left(a_{i 1}, n_{i 1}, \ldots, a_{i t}, n_{i t}\right)\right) \\
& =\partial F_{f g}\left(a_{11}, n_{11}, \ldots, a_{s t}, n_{s t}\right) .
\end{aligned}
$$

Corollary 13.2. The following diagram commutes for any $\mu$-algebra $H$ and for all $n \geq 0, k \geq 1$. In particular this holds if $H$ is a commutative cocommutative K-bialgebra with superproduct:

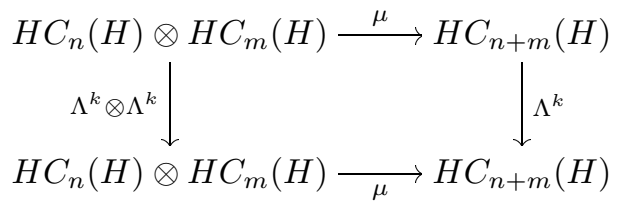

Proof. When $H=K M$ this follows from Theorem 13.1 and the fact that $\lambda^{k}(P \times$ $Q) \cong \lambda^{k} P \times \lambda^{k} Q$ (Proposition 10.2). For the general case we need to verify that both compositions $\mu\left(\Lambda^{k}, \Lambda^{k}\right), \Lambda^{k} \mu$ are given by uniform formulas, i.e., we need to know that the calculations leading to (3) above work for bialgebras. But this just boils down to checking that:

(a) $\left(\alpha_{\#} x * \beta_{\#} y\right) \#=(x * y)^{\#} \circ(\alpha \times \beta)^{*}$,

(b) $\left(\gamma_{\#}(x * y)\right)^{\#}=(x * y)^{\#} \circ \gamma^{*}$.

More generally one needs to check that

$$
f\left(\phi_{\#}^{1} g^{1}\left(x^{1}\right), \ldots, \phi_{\#}^{s} g^{s}\left(x^{s}\right)\right)^{\#}=f\left(g^{1}\left(x^{1}\right), \ldots, g^{s}\left(x^{s}\right)\right)^{\#} \circ f\left(\phi^{1}, \ldots, \phi^{s}\right)^{*} .
$$

But this is exactly Definition 12.3 .

\section{TOpological CARRIERS}

In the last five sections of this paper we will show that the topological definitions of the operators $\mu, \Lambda^{k}$ on $H C_{*}(K M)$ as given in $\S 1$ agree with the algebraic definitions given above. The idea is to show that the continuous maps defined in $\S 1$ are carried by "topological carriers" corresponding to the acyclic carriers used above. This section contains the definition of a topological carrier.

Definition 14.1. Suppose that $\left(X,\left\{\psi_{e}\right\}\right)$ is a CW complex. $\left(\psi_{e}: D^{n} \rightarrow X\right.$ is the characteristic map of the cell $e^{n}$.) Then a category of cells for $X$ is a pair $(\mathcal{E}(X), L)$ where

(a) $\mathcal{E}(X)$ is a small category whose object set is the set of cells of $X$ so that:

(i) Each cell is the source of only finitely many morphisms.

(ii) For each nonidentity morphism $\alpha: e \rightarrow e^{\prime}, \operatorname{dim}(e)>\operatorname{dim}\left(e^{\prime}\right)$.

(b) $L$ is a contravariant functor from $\mathcal{E}(X)$ to the category of finite $\mathrm{CW}$ complexes over $X$ so that: 
(i) The cells of $L(e)$ is in 1-1 correspondence with the morphisms in $\mathcal{E}(X)$ with source $e$. (The cell corresponding to $\alpha: e \rightarrow e^{\prime}$ is denoted $e_{\alpha}$.)

(ii) The map $\pi_{e}: L(e) \rightarrow X$ sends the open cell $e_{\alpha}$ homeomorphically onto the open cell $e^{\prime}$, in fact we assume that $\pi_{e} \circ \psi_{e_{\alpha}}=\psi_{e^{\prime}}$.

(iii) For each $\alpha: e \rightarrow e^{\prime}$ the induced map $\alpha^{*}: L\left(e^{\prime}\right) \rightarrow L(e)$ satisfies $\alpha^{*} \circ \psi_{e_{\beta}}=$ $\psi_{e_{\beta \alpha}}$.

Proposition 14.2. If $(\mathcal{E}(X), L)$ is a category of cells for $X$ then

(a) $X$ has the quotient topology with respect to $\coprod_{e} L(e)$.

(b) If an orientation is chosen for each cell of $X$ then the morphisms of $\mathcal{E}(X)$ of degree -1 acquire incidence numbers $r_{\alpha}$ and $\mathcal{E}(X)$ becomes a basis category.

If $Y$ is any space and $X$ is as above then a topological carrier from $X$ to $Y$ is defined to be a contravariant functor $F$ from $\mathcal{E}(X)$ to the category of weakly contractible spaces over $Y$, i.e., spaces with the weak homotopy type of a point. A continuous map $f: X \rightarrow Y$ is said to be carried by the carrier $F$ if for each cell $e$ in $X$ there is a lifting $\tilde{f}_{e}: L(e) \rightarrow F(e)$ of the composition $L(e) \rightarrow X \rightarrow Y$ so that the following diagram commutes for all morphisms $\alpha: e \rightarrow e^{\prime}$ in $\mathcal{E}(X)$.

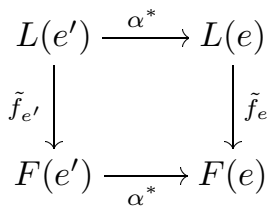

Proposition 14.3. Every topological carrier from $X$ to $Y$ carries at least one map and any two such maps are homotopic.

We say that the topological carrier $F$ is regular if $Y$ and each $F(e)$ is a $\mathrm{CW}$ complex and all maps $F(e) \rightarrow Y$ and $F\left(e^{\prime}\right) \rightarrow F(e)$ are regular in the sense that they send open cells homeomorphically onto open cells. Since a regular map $F(e) \rightarrow Y$ induces a well-defined chain map of cellular chain complexes $C(F(e)) \rightarrow C(Y)$ we get an ordinary carrier " $C(F)$ " from $C(X)$ to $C(Y)$.

Proposition 14.4. Let $F$ be a regular topological carrier from $X$ to $Y$. Then there is a cellular map $f: X \rightarrow Y$ which is carried by $F$ and the induced map $f_{*}: C(X) \rightarrow C(Y)$ is carried by $C(F)$.

There are some CW complexes which have canonical cell categories. We say that a CW complex $X$ is weakly regular if for every pair of open cells $e, e^{\prime}$ the inverse image $\psi_{e}^{-1}\left(e^{\prime}\right) \subseteq D^{n}$ is locally path connected and the map $\psi_{e}^{-1}\left(e^{\prime}\right) \rightarrow e^{\prime}$ is open. In particular this implies that each component of $\psi_{e}^{-1}\left(e^{\prime}\right)$ maps onto $e^{\prime}$ so the closure of each cell is a finite subcomplex of $X$. With a little thought one can see that $\psi_{e}^{-1}\left(e^{\prime}\right)$ has only finitely many components.

Definition 14.5. If $X$ is a weakly regular CW complex then the canonical cell category for $X$ is defined as follows.

(a) The objects of $\mathcal{E}(X)$ are the cells of $X$. A morphism $e^{n} \rightarrow e^{\prime}$ is defined to be a component of $\psi_{e}^{-1}\left(e^{\prime}\right) \subseteq D^{n}$.

(b) For each cell $e^{n}, L(e)$ is defined to be the quotient space of $D^{n}$ modulo the equivalence relation $x \sim y$ if $\psi_{e}(x)=\psi_{e}(y) \in e^{\prime} \subseteq \bar{e}$ and $x, y$ lie in the same component of $\psi_{e}^{-1}\left(e^{\prime}\right)$. This means that each element of $L(e)$ is uniquely represented by a pair $(x, \alpha)$ where $\alpha: e \rightarrow e^{\prime}$ is a morphism in $\mathcal{E}(X)$ and $x \in e^{\prime}$. 
(c) $\pi_{e}: L(e) \rightarrow X$ is given by $\pi_{e}(x, \alpha)=x$ and $\alpha^{*}: L\left(e^{\prime}\right) \rightarrow L(e)$ is given by $\alpha^{*}(x, \beta)=(x, \beta \alpha)$.

(d) The composition of the quotient map $D^{m} \rightarrow L\left(e^{\prime}\right)$ with the map $\alpha^{*}: L\left(e^{\prime}\right) \rightarrow$ $L(e)$ gives the characteristic map from the cell in $L(e)$ corresponding to $\alpha: e \rightarrow e^{\prime}$.

As an example, the geometric realization of a simplicial set $X$ is weakly regular and its canonical cell category resembles the category of simplices of $X$ (without the degeneracies).

\section{The CANONical CELl CATEgory For $V M \simeq B M$}

If $M$ is an associative monoid then let $V M$ be the space of all finite nonempty configurations $X \subseteq S^{1}$ together with labels in $M$. As in the case of $B M$ in $\S 1$ the points in $X$ are allowed to merge with corresponding labels being multiplied in clockwise order. However we do not delete points with resulting label equal to 1.

There is a map $V M \rightarrow B M$ given by ignoring the points in $X$ with label 1 and inserting $1 \in S^{1}$ with label $1 \in M$ if it is not already in the configuration.

Proposition 15.1. $V M \simeq B M$.

Proof. Let $V_{0} M$ be the subspace of $V M$ consisting of all configurations $X$ containing the point $1 \in S^{1}$. Then $V_{0} M$ is homeomorphic to the nondegenerate realization $\|F(M)\|$ of the simplicial set $F(M)$ of $\S 1$ and thus $V_{0} M \simeq B M$.

To see that $V_{0} M \simeq V M$ we note first that there is a retraction $r: V M \rightarrow V_{0} M$ given by inserting the point $1 \in S^{1}$ with label $1 \in M$. A homotopy between $r$ and the identity on $V M$ is given as follows. For any nonempty configuration $X \subseteq S^{1}$ we detach one new point from each point of $X$ with label 1 and move it clockwise until it merges with the next point. When one of these new points passes the point $1 \in S^{1}$ we split it in two and leave a fixed point at 1 with label 1 .

Now we need a cell decomposition of $V M$ so that $V_{0} M$ is a subcomplex. If $x=\left(x_{0}, \ldots, x_{n}\right) \in M^{n+1}$ then let $e_{x} \subseteq V_{0} M$ be the set of all configurations $\left\{y_{0}, \ldots, y_{n}\right\}$ in $S^{1}$ written counterclockwise with $y_{0}=1$ so that $y_{i}$ has label $x_{i}$. The characteristic map $\psi_{e_{x}}: \Delta^{n} \rightarrow V_{0} M$ of the $n$-cell $e_{x}$ is given in barycentric coordinates by $\psi_{e_{x}}(t)=\left\{y_{0}, \ldots, y_{n}\right\}$ where $y_{i}=\exp \left(2 \pi \sqrt{-1}\left(t_{0}+\cdots+t_{i-1}\right)\right)$ with appropriate labels. Then $\eta_{e_{x}}: \partial \Delta^{n} \rightarrow V_{0} M^{n-1}$ is clearly regular so $V_{0} M$ becomes a weakly regular $\mathrm{CW}$ complex.

For each $x=\left(x_{0}, \ldots, x_{n}\right) \in M^{n+1}$ let $u_{x} \subseteq V M$ be the set of all configurations $\left\{y_{0}, \ldots, y_{n}\right\}$ with $y_{n}<1<y_{0}$ in counterclockwise order with labels $x_{i}$. Then $u_{x}$ is an $(n+1)$-cell with characteristic map $\psi_{u_{x}}: \Delta^{n+1} \rightarrow V M$ given by $\psi_{u_{x}}(t)=$ $\left\{y_{0}, \ldots, y_{n}\right\}$ where $y_{i}=\exp \left(2 \pi \sqrt{-1}\left(t_{0}+\cdots+t_{i}\right)\right)$. Again $\eta_{u_{x}}: \partial \Delta^{n+1} \rightarrow V M^{n}$ is regular so $V M$ is a weakly regular $\mathrm{CW}$ complex. Consequently there is a canonical cell category $\mathcal{E}(V M)$. From the geometric description it is obvious that there is one morphism $e_{x} \rightarrow e^{\prime}$ in $\mathcal{E}(V M)$ for every simplex in $\Delta^{n}$ and one morphism $u_{x} \rightarrow e^{\prime \prime}$ for every simplex in $\Delta^{n+1}$. Furthermore the incidence numbers are all \pm 1 .

A morphism $e_{x} \rightarrow e_{y}$ where $y=\left(y_{0}, \ldots, y_{m}\right)$ is given by an epimorphism $\alpha$ : $Z[n] \rightarrow Z[m]$ so that $\bar{\alpha}(0)=0$ (these are dual to monomorphisms $[m] \rightarrow[n]$ in $\Delta$ ) and so that $y=\alpha_{\#} x$. There are no morphisms $e_{x} \rightarrow u_{y}$. A morphism $u_{x} \rightarrow u_{y}$ is given by an epimorphism $\alpha: Z[n] \rightarrow Z[m]$ in $\Delta$ so that $y=\alpha_{\#} x$. A morphism $u_{x} \rightarrow e_{y}$ is given by an epimorphism $\alpha: Z[n] \rightarrow Z[m]$ so that $y=\alpha_{\#} x$ and $\bar{\alpha}^{-1}(0)$ contains either 0 or $n$. 
The incidence numbers for the morphisms in $\mathcal{E}(V M)$ of degree -1 are indicated by the following proposition.

Proposition 15.2. In the cellular chain complex of $V M$ the boundaries of the generators $(n, x), u(n, x)$ corresponding to $e_{x}, u_{x}$ have boundaries:

$$
\begin{gathered}
\partial(n, x)=\sum_{i=0}^{n}(-1)^{i}\left(n-1, \partial_{i} x\right), \\
\partial u(n, x)=-\sum_{i=0}^{n-1}(-1)^{i} u\left(n-1, \partial_{i} x\right)+(n, x)-(-1)^{n}(n, t x) .
\end{gathered}
$$

16. The CANONiCAL CELL CATEgORY FOR $W M \simeq G M$

Let $W M$ denote the space of all pairs $(C, X)$ where $C \in B(1)=\mathbb{C} P^{\infty}$ and $X$ is a finite nonempty configuration in $C$ with labels in $M$. We give $W M$ the topology analogous to that of the previous configuration spaces $V M, B M, G M$. Then $W M$ is a fiber bundle over $B U(1)$ with fiber $V M$. There is a natural map $W M \rightarrow G M$ given by ignoring points with label 1 . Since $V M \simeq B M$ we conclude the following.

Theorem 16.1. $W M \simeq G M$.

To construct a cell decomposition for $W M$ we need to start with the standard cell decomposition of $B U(1)=\mathbb{C} P^{\infty}$ which has one cell $e^{2 a}$ in every even dimension so that $e^{0} \cup e^{2} \cup \cdots \cup e^{2 a}=\mathbb{C} P^{a}$. Thus the open cells are given by $e^{2 a}=\left\{\left[z_{0}, \ldots, z_{a}\right] \in \mathbb{C} P^{a} \mid z_{a} \neq 0\right\}$. The characteristic map $\psi_{2 a}: D^{2 a} \rightarrow \mathbb{C} P^{a}$ of $e^{2 a}$ is given by $\psi_{2 a}\left(z_{0}, \ldots, z_{a-1}\right)=\left[z_{0}, \ldots, z_{a-1}, x\right]$ where $x=\sqrt{1-\|z\|^{2}}$ is the unique nonnegative real number so that $\left(z_{0}, \ldots, z_{a-1}, x\right) \in S^{2 a+1}$. This has an obvious lifting $\tilde{\psi}_{2 a}: D^{2 a} \rightarrow S^{2 a+1}$ given by $\tilde{\psi}_{2 a}\left(z_{0}, \ldots, z_{a-1}\right)=\left(z_{0}, \ldots, z_{a-1}, x\right)$.

We will use the notation $\not$ to denote the unique element $\phi=\left(z_{0}, \ldots, z_{a}\right)$ of each fiber of $E(1)=S^{\infty} \rightarrow B U(1)=\mathbb{C} P^{\infty}$ so that the last nonzero coordinate $z_{a}$ is positive and real. This is the same as saying that $\not$ lies in the image of $\tilde{\psi}_{2 a}\left(\right.$ int $\left.D^{2 a}\right)$ for some $a \geq 0$.

For each $a, n \geq 0$ and $x \in M^{n+1}$ let $e S_{a}(n, x)$ denote the set of all $(C, X) \in W M$ so that $C \in B U(1)$ lies in the open cell $e^{2 a}$ and $\phi \in X=\left\{y_{0}, \ldots, y_{n}\right\}$ with elements numbered counterclockwise with $y_{0}=\not$ and with corresponding labels $x_{0}, \ldots, x_{n}$. Then $e S_{a}(n, x)$ is an $(n+2 a)$-cell with characteristic map $\psi: D^{2 a} \times \Delta^{n} \rightarrow W M$ given by $\psi(z, t)=\left(\psi_{2 a}(z),\left\{y_{0}, \ldots, y_{n}\right\}\right)$ where $y_{i}=\exp \left(2 \pi \sqrt{-1}\left(t_{0}+\cdots+t_{i-1}\right)\right) \tilde{\psi}_{2 a}(z)$.

Similarly let $e R_{a}(n, x)$ denote the set of all $(C, X) \in W M$ so that $C \in e^{2 a}$ but $\phi \notin X=\left\{y_{0}, \ldots, y_{n}\right\}$ with corresponding labels $x_{0}, \ldots, x_{n}$. Then $e R_{a}(n, x)$ is an $(n+2 a+1)$-cell with characteristic map $\psi: D^{2 a} \times \Delta^{n+1} \rightarrow W M$ given by $\psi(z, t)=\left(\psi_{2 a}(z),\left\{y_{0}, \ldots, y_{n}\right\}\right)$ where $y_{i}=\exp \left(2 p i \sqrt{-1}\left(t_{0}+\cdots+t_{i}\right)\right) \tilde{\psi}_{2 a}(z)$.

Proposition 16.2. This defines a weakly regular cell decomposition of $W M$. The boundary map in the associated cellular chain complex is given by

$$
\begin{gathered}
\partial S_{a}(n, x)=\sum_{i=0}^{n}(-1)^{i} S_{a}\left(n-1, \partial_{i} x\right)+\sum_{i=0}^{n}(-1)^{n i} R_{a-1}\left(n, t^{i} x\right) \\
(\text { i.e. } \partial=b+N),
\end{gathered}
$$




$$
\begin{gathered}
\partial R_{a}(n, x)=-\sum_{i=0}^{n-1}(-1)^{i} R_{a}\left(n-1, \partial_{i} x\right)+S_{a}(n, x)-(-1)^{n} S_{a}(n, t x) \\
\left(\text { i.e., } \partial=-b^{\prime}+1-t\right),
\end{gathered}
$$

where $S_{a}(n, x) \in C_{n+2 a}(W M)$ and $R_{a}(n, x) \in C_{n+2 a+1}(W M)$ are the generators corresponding to $e S_{a}(n, x)$ and $e R_{a}(n, x)$ and the undefined terms $R_{-1}\left(n, t^{i} x\right)$ are understood to be zero.

The canonical cell category $\mathcal{E}(W M)$ can be described as follows. For $A, B=$ $S$ or $R$ there is a morphism $e A_{a}(n, x) \rightarrow e B_{b}(m, y)$ for every epimorphism $\alpha$ : $Z[n] \rightarrow Z[m]$ so that $y=\alpha_{\#} x$ provided that the open cell $e B_{b}(m, y)$ is contained in the closure of $e A_{a}(n, x)$. This geometric condition is equivalent to the following combinatorial conditions.

(a) $b \leq a$ in all cases,

(b) $b<a$ if $(A, B)=(S, R)$.

(c) Suppose that $a=b$ then (as in the case of $V M$ ):

(i) $\bar{\alpha}(0)=0$ in the case $(A, B)=(S, S)$,

(ii) $\bar{\alpha}^{-1}(0)$ contains either 0 or $n$ in the case $(A, B)=(R, S)$,

(iii) $\alpha:[n] \rightarrow[m]$ is a morphism in $\Delta$ in the case $(A, B)=(R, R)$.

This construction generalizes to the following.

Definition 16.3. If $X$ is any cyclic set let $\mathcal{A}(X)$ be the basis category defined as follows.

(1) The objects of $\mathcal{A}(X)$ are symbols $S_{a}(n, x), R_{a}(n, x)$ where $a, n \geq 0$ and $x \in X_{n}$.

(2) $\operatorname{deg} S_{a}(n, x)=n+2 a, \operatorname{deg} R_{a}(n, x)=n+2 a+1$.

(3) A morphism $A_{a}(n, x) \rightarrow B_{b}(m, y)$ is an epimorphism $\alpha: Z[n] \rightarrow Z[m]$ so that $y=\alpha_{*} x$ and the combinatorial conditions (a), (b), (c) above are satisfied.

(4) The incidence coefficients are as indicated in Proposition 16.2.

With this definition we have $\mathcal{E}(W M)=\mathcal{A}\left(M^{\Lambda}\right)$.

Proposition 16.4. For any cyclic set $X$ there is a natural chain homotopy equivalence $\nu_{X}: K \mathcal{B}(X) \stackrel{\sim}{\longrightarrow} K \mathcal{A}(X)$ which is carried by the acyclic carrier which sends $S_{a}(n, x)$ to $K \mathcal{A}(X Z[n])$ which maps to $K \mathcal{A}(X)$ by the cyclic map $x^{\#}: X Z[n] \rightarrow X$ given by $x^{\#}(\alpha: Z[n] \rightarrow Z[k])=\alpha_{*}(x) \in X_{k}$.

Proof. $\tilde{\nu}_{X}\left(S_{a}(n, x)\right)=S_{a}(n, 1)+\sum_{i=0}^{n}(-1)^{n i} U_{a-1}\left(n+1, s t^{i}\right)$.

\section{A topological CARRIER For $\mu: W M \times W M \rightarrow W M$}

We are now ready to show that the topological and algebraic definitions of the multiplication $\mu$ in $H C_{*}(K M)$ agree. We are assuming that $M$ is a commutative monoid with a bimultiplicative pairing $*: M \times M \rightarrow M$ so that $1 * x=x * 1=1$ for all $x \in M$.

Let $\mu: W M \times W M \rightarrow W M$ be given by $\mu((C, X),(D, Y))=(C D, X * Y)$ as in $\S 1$. Then we will construct a regular topological carrier $F_{\mu}$ for $\mu$. For each cell $e\left(A_{a}(n, x), B_{b}(m, y)\right)=e A_{a}(n, x) \times e B_{b}(m, y)$ in $W M \times W M$ where $A, B$ represent either $S$ or $R$ let $F_{\mu}\left(e\left(A_{a}(n, x), B_{b}(m, y)\right)\right)=F_{\mu}(n, m)$ be the space of all pairs $(C, \phi)$ where $C \in B U(1)$ and $\phi: Z[n, m] \rightarrow C$ is a morphism of cyclically ordered sets with the map $(x * y)^{\#}: F_{\mu}(n, m) \rightarrow W M$ given by $(x * y)(C, \phi)=\left(C, \phi_{\#}(x * y)\right)$ 
where $\phi_{\#}(x * y)$ is the image of $\bar{\phi}$ together with labels $\prod\left\{x_{i} * y_{j} \mid \bar{\phi}(i, j)=z\right\}$ for each $z \in \operatorname{Im} \bar{\phi}$.

For any morphism $(\alpha, \beta): e\left(A_{a}(n, x), B_{b}(m, y)\right) \rightarrow e\left(X_{a^{\prime}}\left(n^{\prime}, \alpha_{\#} x\right), Y_{b^{\prime}}\left(m^{\prime}, \beta_{\#} y\right)\right)$ given by two morphisms $\alpha: Z[n] \rightarrow Z\left[n^{\prime}\right], \beta: Z[m] \rightarrow Z\left[m^{\prime}\right]$ let $(\alpha, \beta)^{*}: F_{\mu}\left(n^{\prime}, m^{\prime}\right)$ $\rightarrow F_{\mu}(n, m)$ be given by $(\alpha, \beta)^{*}(C, \phi)=(C, \phi \circ(\alpha \times \beta))$.

Since $F_{\mu}(n, m)$ is clearly contractible we have:

Lemma 17.1. $F_{\mu}$ is a topological carrier from $W M \times W M$ to $W M$.

Lemma 17.2. $\mu: W M \times W M \rightarrow W M$ is carried by $F_{\mu}$.

Proof. Let $\tilde{\mu}: L\left(e A_{a}(n, x)\right) \times L\left(e B_{b}(m, y)\right) \rightarrow F_{\mu}(n, m)$ be given by

$$
\tilde{\mu}((C, X, \alpha),(D, Y, \beta))=(C D, \phi)
$$

where we view $\alpha, \beta$ as morphisms $\alpha: Z[n] \rightarrow C, \beta: Z[m] \rightarrow D$ with images $X, Y$ having labels $\alpha_{\#} x, \beta_{\#} y$ and $\phi: Z[n, m] \rightarrow C D$ is given by $\tilde{\phi}(i, j, k)=$ $(\tilde{\alpha}(i, 0), \tilde{\beta}(j, k), k)$. The following discussion shows that $\tilde{\mu}$ is a continuous function.

Let $\nu: Z[0,1) \times Z[0,1) \rightarrow Z[0,1)$ be the morphism of cyclically ordered sets given by

$$
\nu(x, y, n)= \begin{cases}(x+y, n) & \text { if } x+y<1 \\ (x+y-1, n+1) & \text { if } x+y \geq 1\end{cases}
$$

Then $\nu$ is rotationally invariant in the sense that $\nu \circ\left(T_{a} \times T_{b}\right)=T_{a+b} \circ \nu$ for all $a, b \in \mathbb{R}$ where $T_{a}: Z[0,1) \rightarrow Z[0,1)$ is given by $\widetilde{T}_{a}(x)=x+a$ (if we identify $\widetilde{Z}[0,1)$ with $\mathbb{R})$.

Since $\nu$ is rotationally invariant it induces a morphism $\nu: C \times D \rightarrow C D$ for any $C, D \in B U(1)$. Furthermore the underlying set map $\tilde{\nu}$ of $\nu$ varies continuously with $(C, D) \in B U(1) \times B U(1)$. The map $\phi: Z[n, m] \rightarrow C D$ factors as $\phi=\nu \circ(\alpha \times \beta):$ $Z[n, m] \rightarrow C \times D \rightarrow C D$ so it varies continuously with $((C, X, \alpha),(D, Y, \beta))$.

Lemma 17.3. $F_{\mu}$ is a regular topological carrier and the canonical cell category of $\mathcal{E}\left(F_{\mu}(n, m)\right)$ is naturally isomorphic to $\mathcal{A}(X Z[n, m])$.

Proof. First we need a cell decomposition of $F_{\mu}(n, m)$. For each morphism $\phi$ : $Z[n, m] \rightarrow Z[k]$ and $a \geq 0$ let $e S_{a}(k, \phi)$ be the space of all pairs $(C, \alpha \circ \phi)$ where $C \in e^{2 a} \subseteq B U(1)$ and $\alpha: Z[k] \rightarrow C$ is a morphism so that $\bar{\alpha}(0)=\not$. Let $e R_{a}(k, \phi)$ be the space of all pairs $(C, \beta \circ \phi)$ where $C \in e^{2 a}$ and $\beta: Z[k] \rightarrow C$ is a morphism so that $\not<<\beta(0)<\cdots<\beta(k)<\sigma(\phi)$.

The characteristic maps

$$
\psi: D^{2 a} \times \Delta^{n}, D^{2 a} \times \Delta^{n+1} \rightarrow F_{\mu}(n, m)
$$

for $e S_{a}(k, \phi), e R_{a}(k, \phi)$ are given by $\psi(z, t)=\left(\psi_{2 a}(z), \alpha \circ \phi\right),\left(\psi_{2 a}(z), \beta \circ \phi\right)$ respectively where $\alpha, \beta$ are given by $\bar{\alpha}(i)=\exp \left(2 \pi \sqrt{-1}\left(t_{0}+\cdots+t_{i-1}\right)\right) \tilde{\psi}_{2 a}(z)$ and $\bar{\beta}(j)=\exp \left(2 \pi \sqrt{-1}\left(t_{0}+\cdots+t_{j}\right)\right) \tilde{\psi}_{2 a}(z)$.

By comparing formulas it is clear that the maps $(x * y)^{\#}: F_{\mu}(n, m) \rightarrow W M$ and $(\alpha \times \beta)^{*}: F_{\mu}\left(n^{\prime}, m^{\prime}\right) \rightarrow F_{\mu}(n, m)$ are all regular. It follows that $F_{\mu}(n, m)$ is weakly regular and that $(x * y)^{\#}$ induces a morphism of basis categories. This forces $\mathcal{E}\left(F_{\mu}(n, m)\right)$ to be isomorphic to $\mathcal{A}(X Z[n, m])$. 
Theorem 17.4. The topological and algebraic definitions of the multiplication

$$
\mu: H C_{*}(K M) \otimes H C_{*}(K M) \rightarrow H C_{*}(K M)
$$

agree.

Proof. The regular topological carrier $F_{\mu}$ gives an operation $\mu_{A}$ on $K \mathcal{A}\left(M^{\Lambda}\right)$. The acyclic carrier $E$ gives an operation $\mu_{B}$ on $K \mathcal{B}\left(M^{\Lambda}\right)=T(K M)$. Thus it suffices to show that the following diagram commutes up to homotopy where $\nu$ is the chain homotopy equivalence given by Proposition 16.4.

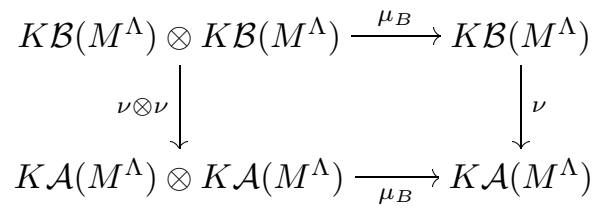

In order to do this we form the mapping cylinder $C y l(\nu)$ of $\nu$ and construct an acyclic carrier for a map $\mu_{C}: C y l(\nu) \otimes C y l(\nu) \rightarrow C y l(\nu)$ making the following diagram commute.

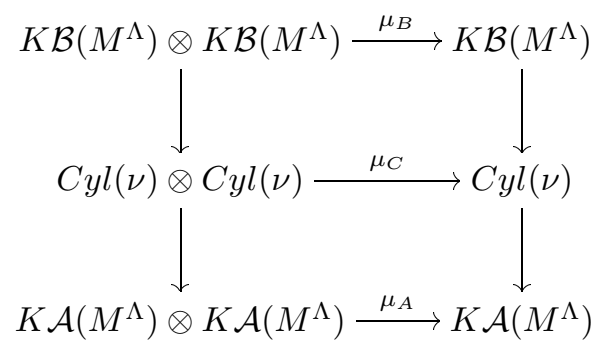

The mapping cylinder $C y l(\nu)$ is defined to be $K \mathcal{C}\left(M^{\Lambda}\right)$ where $\mathcal{C}(X)$ is the basis category defined for any cyclic set $X$ as follows.

The category $\mathcal{C}(X)$ is a union of three disjoint full subcategories $\mathcal{A}(X), \mathcal{B}(X)$, and $\sum \mathcal{B}(X)$ where $\sum \mathcal{B}(X)$ is the same as $\mathcal{B}(X)$ except that the degrees of all objects are raised by 1 and the incidence coefficients change in sign $\left(\operatorname{deg} \sum A=\right.$ $\left.\operatorname{deg} A+1, r_{\sum \alpha}=-r_{\alpha}\right)$. There are no morphism between $\mathcal{A}(X)$ and $\mathcal{B}(X)$ nor from $\mathcal{A}(X) \amalg \mathcal{B}(X)$ into $\sum \mathcal{B}(X)$.

The morphisms from $\sum \mathcal{B}(X)$ to $\mathcal{B}(X)$ are simply morphisms in $\mathcal{B}(X)$. Thus $\mathcal{C}(X)\left(\sum A, B\right)=\mathcal{B}(X)(A, B)$ for all $A, B$ in $\mathcal{B}(X)$. The identity morphisms $\sum A \rightarrow$ $A$ have incidence coefficient equal to 1 . All other morphisms $\sum A \rightarrow B$ have coefficient zero. The morphisms from $\sum S_{a}(n, x) \in \sum \mathcal{B}(X)$ to $A_{b}(m, y) \in \mathcal{A}(X)$ $(A=r$ or $S)$ are defined to be the morphism $\alpha: Z[n] \rightarrow Z[m]$ so that $y=\alpha_{\#} x$. The only morphisms from $\mathcal{B}(X)$ to $\mathcal{A}(X)$ with nonzero incidence coefficient are those which occur in the formula for $\nu_{X}$. These are $i d: \sum S_{a}(n, x) \rightarrow S_{a}(n, x)$ with coefficient 1 and $s t^{i}: \sum S_{a}(n, x) \rightarrow R_{a-1}\left(n+1, s t^{i} x\right)$ with coefficient $(-1)^{n i}$ for $0 \leq i \leq n$. Composition is defined in the obvious way by composition of morphisms in $\mathcal{C}$ os.

The inclusion functors $\mathcal{A}(X) \rightarrow \mathcal{C}(X)$ and $\mathcal{B}(X) \rightarrow \mathcal{C}(X)$ induce the chain maps from $K \mathcal{A}(X), K \mathcal{B}(X)$ into $C y l(\nu)=K \mathcal{C}(X)$ which appear in (0) above for 
$X=M^{\Lambda}$. To prove the theorem it now suffices to construct an acyclic carrier for a map $\mu_{C}: K \mathcal{C}(X) \otimes K \mathcal{C}(X) \rightarrow K \mathcal{C}(X)$ which is compatible with $\mu_{A}, \mu_{B}$. Such an acyclic carrier is given by sending $\left(A_{a}(n, x), B_{b}(m, y)\right)$ in $\mathcal{C}\left(M^{\Lambda}\right) \times \mathcal{C}\left(M^{\Lambda}\right)$ for $A, B=R, S, \sum S$ to the complex $K \mathcal{C}(X Z[n, m])$ which maps to $K \mathcal{C}\left(M^{\Lambda}\right)$ by the cyclic map $(x * y)^{\#}: X Z[n, m] \rightarrow M^{\Lambda}$.

\section{A TOPOLOGICAL CARRIER FOR $\Lambda^{k}: W M \rightarrow W M$}

Theorem 18.1. The topological and algebraic definitions of the power operations $\Lambda^{k}=1 / k \Psi^{k}$ on $H C_{*}(K M)$ agree.

Remark. This result is essentially due to Goodwillie since our topological definitions of $\Lambda^{k}$ is just a rewording of this intuitive description of these operations.

Proof. This proof is analogous to, but easier than, the proof of Theorem 17.4. To minimize repetition we give only the first half of the proof.

Let $\Lambda^{k}: W M \rightarrow W M$ be the continuous function $\Lambda^{k}(C, X)=\left(C^{k}, \Lambda^{k}(X)\right)$. Then a regular topological carrier $F_{\Lambda}$ for $\Lambda^{k}$ can be given as follows. For any cell $e A_{a}(n, x)$ in $W M$ where $A=R$ or $S$ let $F_{\Lambda}\left(e A_{a}(n, x)=F_{\Lambda}(n)\right.$ be the space of all pairs $(C, \phi)$ where $C \in B U(1)$ and $\phi: \lambda^{k} Z[n] \rightarrow C$ is a morphism of cyclically ordered sets with the map $x^{\#}: F_{\Lambda} \rightarrow W M$ given by $x^{\#}(C, \phi)=\left(C, \phi_{\#} x\right)$. Any morphism $\alpha: e A_{a}(n, x) \rightarrow e B_{b}(m, y)$ is given by a morphism $\alpha: Z[n] \rightarrow Z[m]$ so that $y=\alpha_{\#} x$. Let $\alpha^{*}: F_{\Lambda}(m) \rightarrow F_{\Lambda}(n)$ be given by $\alpha^{*}(C, \phi)=\left(C, \phi \circ \lambda^{k} \alpha\right)$.

To see that $\Lambda^{k}$ is carried by $F_{\Lambda}$ let $\widetilde{\Lambda}^{k}: L\left(e A_{a}(n, x)\right) \rightarrow F_{\Lambda}(n)$ be given by $\widetilde{\Lambda}^{k}((C, X), \alpha)=\left(C^{k}, \phi\right)$ where $\phi: \lambda^{k} Z[n] \rightarrow C^{k}$ is the composition of $\lambda^{k} \alpha:$ $\lambda^{k} Z[n] \rightarrow \lambda^{k} C$ and the unique continuous morphism $\rho: \lambda^{k} C \rightarrow C^{k}$ so that $\bar{\rho}(x)=x^{k}$.

The remainder of the proof is analogous to the proof for $F_{\mu}$, for example, in the final step we use the acyclic carrier which sends $A_{a}(n, x) \in \mathcal{C}\left(M^{\Lambda}\right)$ for $A=$ $R, S, \sum S$ to the complex $K \mathcal{C}\left(X \lambda^{k} Z[n]\right)$ which maps to $K \mathcal{C}\left(M^{\Lambda}\right)$ by $x^{\#}$. This defines a chain map $\Lambda_{C}^{k}: K \mathcal{C}\left(M^{\Lambda}\right) \rightarrow K \mathcal{C}\left(M^{k}\right)$ compatible with the topologically defined operators $\Lambda_{A}^{k}$ on $K \mathcal{A}\left(M^{\Lambda}\right)$ and the algebraically defined operators $\Lambda_{B}^{k}$ on $K \mathcal{B}\left(M^{\Lambda}\right)$.

\section{REFERENCES}

[C] A. Connes, Noncommutative differential geometry, Publ. IHES 62 (1985), 41-144. MR 87i:58162

[FT] B. Feigin and B. Tsygan, Additive K-theory, K-Theory, Arithmetic and Geometry, Lecture Notes in Math. 1289, Springer, New York-Heidelberg-Berlin, 1987, pp. 67-209. MR 89a: 18017

[Ga] P. Gaucher, Opérations sur l'homologie d'algèbres de matrices et homologie cyclique, Ph.D. thesis, Universite Louis Pasteur, Strasbourg, France, 1992.

[Go] T. Goodwillie, Cyclic homology, derivatives and the free loop space, Topology 24 (1985), 187-215. MR 87c:18009

[H] D. Husemoller, Homology of certain $H$-spaces as group ring objects, Algebra, Topology and Category Theory, A collection of papers in honor of Samuel Eilenberg, Academic Press, New York-San Francisco-London, 1976. MR 54:1222

[IK] K. Igusa and J. Klein, The Borel regulator map on pictures II: an example from Morse theory, preprint.

[IZ] K. Igusa and D. Zacharia, On the cyclic homology of monomial relation algebras, J. Algebra 151 (1992), 502-521. MR 93k:19005

$[\mathrm{J}] \quad$ J. Jones, Cyclic homology and equivariant homology, Invent. Math. 87 (1987), 403-423. MR 88f: 18016 
[L] J.-L. Loday, Opérations sur l'homologie cyclique des algèbres commutatives, Invent. Math. 96 (1989), 205-230. MR 89m:18017

[LQ] J.-L. Loday and D. Quillen, Cyclic homology and the Lie algebra homology of matrices, Comment. Math. Helv. 59 (1984), 565-591. MR 86i:17003

[S] M. E. Sweedler, Hopf algebras, Benjamin, New York, 1969. MR 40:5705

[W] F. Waldhausen, Algebraic K-theory of spaces, Lecture Notes in Math. 1126, Springer, 1985, pp. 318-419. MR 86m:18011

Department of Mathematics, Brandeis University, Waltham, Massachusetts 022549110 